\title{
Statistical Modeling of Bowing Control Applied to Violin Sound Synthesis
}

\author{
Esteban Maestre, Merlijn Blaauw, Jordi Bonada, Enric Guaus, and Alfonso Pérez
}

\begin{abstract}
Excitation-continuous music instrument control patterns are often not explicitly represented in current sound synthesis techniques when applied to automatic performance. Both physical model-based and sample-based synthesis paradigms would benefit from a flexible and accurate instrument control model, enabling the improvement of naturalness and realism. We present a framework for modeling bowing control parameters in violin performance. Nearly non-intrusive sensing techniques allow for accurate acquisition of relevant timbre-related bowing control parameter signals. We model the temporal contour of bow velocity, bow pressing force, and bow-bridge distance as sequences of short Bézier cubic curve segments. Considering different articulations, dynamics, and performance contexts, a number of note classes are defined. Contours of bowing parameters in a performance database are analyzed at note-level by following a predefined grammar that dictates characteristics of curve segment sequences for each of the classes in consideration. As a result, contour analysis of bowing parameters of each note yields an optimal representation vector that is sufficient for reconstructing original contours with significant fidelity. From the resulting representation vectors, we construct a statistical model based on Gaussian mixtures suitable for both the analysis and synthesis of bowing parameter contours. By using the estimated models, synthetic contours can be generated through a bow planning algorithm able to reproduce possible constraints caused by the finite length of the bow. Rendered contours are successfully used in two preliminary synthesis frameworks: digital waveguide-based bowed string physical modeling and sample-based spectral-domain synthesis.
\end{abstract}

Index Terms-Audio systems, Gaussian distributions, gesture modeling, music, pattern classification, signal synthesis, violin bowing control.

\section{INTRODUCTION}

$\mathbf{T}$ HE complexity of control in musical instrument performance stands out when dealing with excitation-continuous or sustained instruments (e.g., bowed-strings or winds). In contrast to the case of excitation-instantaneous musical instruments (e.g., drums or piano-like instruments), variations of sound are achieved by continuous modulations of the physical actions (or controls) directly involved in sound production mechanisms, i.e., instrumental gestures [1]. Difficulties in

Manuscript received March 31, 2009; revised November 28, 2009. Current version published April 14, 2010. This work was supported in part by Yamaha Corporation and in part by the EU-ICT Project SAME. Part of this work was carried out at the Center for Computer Research in Music and Acoustics (CCRMA, Stanford University), during a research stay supported in part by the Agència de Gestió dAjuts Universitaris i de Recerca (AGAUR) through a BE Pre-doctoral Program scholarship. The associate editor coordinating the review of this manuscript and approving it for publication was Dr. Federico Fontana.

The authors are with the Music Technology Group, Universitat Pompeu Fabra, 08002 Barcelona, Spain (e-mail: esteban.maestre@upf.edu).

Digital Object Identifier 10.1109/TASL.2010.2040783

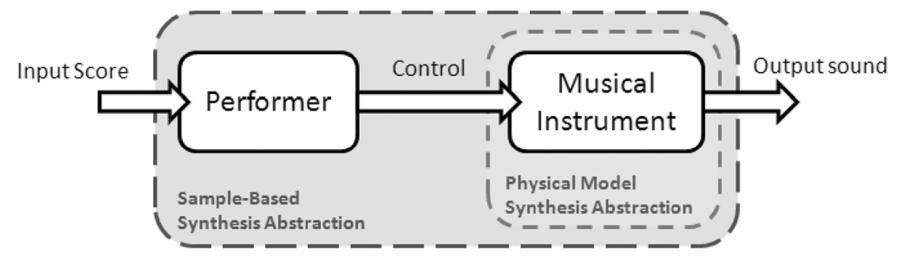

Fig. 1. Simplified diagram of musical instrument sound production in human performance. Abstractions made boby physical modeling synthesis and samplebased synthesis are illustrated.

quantitatively understanding the continuous nature of such controls [2] have prevented excitation-continuous instrumental sound synthesis from achieving a greater success.

Fig. 1 shows a simplified diagram of musical instrument sound production by human performance. In general, a performer transforms the sequence of discrete events appearing in a musical score into the continuous physical actions (or controls) needed for the instrument to produce the desired output sound. While the important role of the performer appears to be crucial in musical performance, it is usual to find sound synthesizers (either based on physical models, or on sample processing) missing an explicit representation of the control carried out by the musician.

In particular, the abstraction taking place in physical modelbased synthesis places the focus on the sound production mechanisms of the instrument. Even though the flexibility of control tends to be preserved, the lack of appropriate control parameter signals represents an important drawback for use in automatic performance. For the case of sample-based sound synthesis, the abstraction usually includes the performer by pairing score annotations wisound recordings. Although higher sound fidelity might be achieved (actual sound recordings are used as source material), flexibility limitations and possible timbre discontinuities often make it difficult to get the feeling of natural performance.

Withe aim of improving the naturalness of synthetic sound in excitation-continuous musical instruments, modeling instrumental control patterns should provide a means for abstracting a proper representation of the performer. Indeed, an explicit decoupling from the instrument would free instrumental sound modeling from control-based design constraints that traditionally appear in offline synthesis scenarios (e.g., NoteOn-like events triggering sound rendering).

The training process in traditional musical instrument performance is based on a technique of continual practice. For that reason, a learning-by-example approach involving the acquisition of instrumental gesture parameters appears to be 
more suited for constructing a quantitative model able to emulate control habits in excitation-continuous musical instrument performance. This paper addresses bowing control in violin performance. A statistical model is first constructed making use of instrumental gesture data acquired from real performance, and then applied for rendering synthetic bowing controls from written scores. Generated bowing parameter signals are finally used for driving sound synthesis.

\section{A. Analysis of Bowing Control Parameters}

Because of the complex and continuous nature of physical actions involved in the control of bowed-string instruments, the acquisition and analysis of bowed-string instrumental gestures has been an active and challenging topic of study for several years, and has led to diverse successful approaches.

In the work presented by Askenfelt [3], [4], a thin resistance wire is inserted into the bow hair in conjunction wielectrified strings in order to detect the contact position. For the bow pressure, four strain gages (two at the tip and two at the frog) are used. Later, Paradiso and Gershenfeld [5] measured bow transversal position by means of oscillators driving antennas (electric field sensing), and bow pressure by using a force-sensitive resistor below the forefinger. Recent implementations resulted in less intrusive systems. In the approach presented by Young [6], bow pressing force is deduced by measuring downward and lateral bow stick deformation using foil strain gages, while bow position wirespect to the bridge is measured in a similar way to that of [5]. Data are sent to a computer via a wireless transmitter mounted at the frog. Rasamimanana and colleagues [7] perform wireless measurements of bow acceleration by means of accelerometers attached to the bow. Bow velocity profiles are reconstructed by using video data in a postprocessing step [8]. Accuracy and robustness in bow force measurement were recently taken to a higher level (see [9], [10] and a re-implementation in [11]) by using strain gages attached to the frog end of the hair ribbon, thus measuring ribbon deflection. In a more recent approach presented in [12], bowing parameters were very accurately measured by means of a commercially available electromagnetic field-based tracking device. Later, this methodology based on tracking positions of string ends and hair ribbon ends was adapted to a more expensive and commercial camera-based motion capture system that needed boa more complex calibration process (using many markers), and a noise reduction postprocessing step [13].

Given the availability of bowing control data, several works have recently presented techniques for quantitative analysis. Rasamimanana and coworkers [7] used bow acceleration extrema for automatic bow stroke classification by applying linear discriminant analysis to a number of features extracted from the acceleration contour. Later, authors continued their work [14] presenting an analysis of performer arm coordination (joint angles) under different bow stroke frequencies. Young [15] extended automatic bowing technique classification to a larger number of bowing techniques across different performers by extracting the principal components of raw bow acceleration and measurement data coming from a strain gage sensor attached to the bow. Recently, a very interesting work [16] approaches the analysis of bow velocity contours by describing motion from a kinetic perspective based on effort, allowing for the observation of anticipation effects.

\section{B. Synthesis of Bowing Control Parameters}

The challenging problem of synthesizing bowing parameters from a musical score has also been addressed in past studies. A first attempt is found in an early work by Chafe [17], where he presented an algorithm for rendering a number of violin performance gesture parameter contours (including boleft- and righthand parameters) by concatenating short segments following a number of handmade rules. Extensions to this approach using left hand articulations and string changes were introduced by Jaffe and Smi[18] for controlling a digital waveguide bowedstring physical model. Although these methods result in an interesting application of the analysis of certain human performance habits and/or conceptions, boapproaches lack real data-driven definition of segment contours parameters. Similarly, some research was pursued using standard ADSR envelopes MIDI-controlled synthesis of the violin, exposing the limitations of the chosen contour representation [19]. A very recent and interesting study incorporating real data is found in [9], where bow velocity and bow force contours of different bow strokes are quantitatively characterized and reconstructed using mostly sinusoidal segments. The author considers different dynamics and bow strokes (mostly focused on isolated notes), but flexibility limitations of the proposed contour representation prevent the model from generalizing its application to other more sustained bowing techniques not based solely on bow strokes (e.g., longer détaché notes or legato articulations).

Previously, author in [2] pointed out directions towards a general framework for the automatic characterization of real instrumental gesture parameter contours using sequences of parametric Bézier curves, foreseeing them as a more powerful and flexible basis for representation and modeling of real data contour shapes (see their application to speech prosody modeling in [20]). It was aimed at providing a means for reconstructing contours by concatenating short curve units in a similar manner as introduced in [17] and [18], but introduced a structured notelevel representation (as opposed to the work presented by Battey [21] dealing with audio perceptual attributes). Later, in a first quantitative application of a note-level structured representation similar to the technique proposed in this work, authors in [22] used concatenated Bézier curves for pursuing a model of different articulations in singing voice performance using pitch and energy contours.

\section{Violin Sound Synthesis}

Capture or modeling of bowing control applied to sound synthesis has traditionally been more present in the context of physical models, often centered around the digital waveguide modeling framework introduced by Smith [23]. Serafin and Young [24] successfully evaluated a real-time application combining a bowing control measurement system [6] with Smith's digital waveguide bowed string model featuring a friction model [25]. For the case of offline scenarios, works in [17]-[19] pursue the application of synthetic bowing controls to offline synthesis, but none of the models are obtained from real performance data. Conversely, Demoucron [9] uses real bowing control data for 
successfully reconstructing bow velocity and bow force contours of a number of isolated bow strokes.

For the case of sample-based approaches, Schoner and colleagues carried out research in real-time cross-synthesis, driven by acquired bowing parameters [26]. Although it was an important first step towards improving the flexibility of samplebased synthesis, no synthetic control parameters were used. In general, synthetic bowing control is still a missing component in sample-based violin sound synthesis. While extended commercial sample-based systems feature a vast number of possibilities in terms of articulation, pitch, etc. (e.g., Vienna Symphonic Library 1 ), sample concatenation and control flexibility limitations often represent a noticeable drawback when it comes to naturalness of synthetic sound. Specialized spectral domain sound transformations oriented towards real-time expressivity control using traditional MIDI-based keyboard interfaces can also be found in the market. Both the Garritan Solo Stradivari ${ }^{2}$ and the Synful Orchestra ${ }^{3}$ [27] achieve such sample transformations. An interesting feature of Garritan is that it incorporates a sample database of string vibration signals where, following sample transformation and concatenation, the body resonances are added by convolution with an estimated impulse response. It is worth noting that Synful is a modeling component able to synthesize spectral envelopes from time-varying perceptual attribute signals created from an input score.

\section{Outline}

In this paper, we present a general and extensible framework for modeling contours of bowing control parameters (bow velocity, bow force, and bow-bridge distance) for different articulations and performance contexts by using concatenated Bézier cubic curves. Two of the primary advantages of this modeling framework are its ability to represent contours with fidelity and robustness and its use of curve parameter extraction that is carried out automatically from real performance data (see Sections II and III for details on data acquisition and analysis of bowing control data), providing a representation suitable for both bowing control analysis and synthesis (see Section IV for details on a statistical model estimated from the curve parameters). We provide details concerning its application to rendering bowing parameter contours from an input score (Sections V and VI) and outline the application of synthetic contours of bowing parameters for controlling both a digital waveguide physical model (see Section VII-B) and a sample-based spectral domain concatenative synthesizer (see Section VII-C).

The main components of a violin sound synthesis scenario utilizing the presented modeling framework are sketched in Fig. 2. From an input score, bowing control models obtained from real data are used for generating synthetic contours. In one portion of the schematic, synthetic bowing parameters are directly used to drive a physical model, where the string vibration synthetic signal is convolved with a violin body impulse response obtained from real data. In the other portion, rendered contours are used both in the sample retrieval and sample

\footnotetext{
${ }^{1}$ http://vsl.co.at.

${ }^{2} \mathrm{http}: / / \mathrm{www}$.garritan.com/stradivari.html.

${ }^{3}$ http://www.synful.com.
}

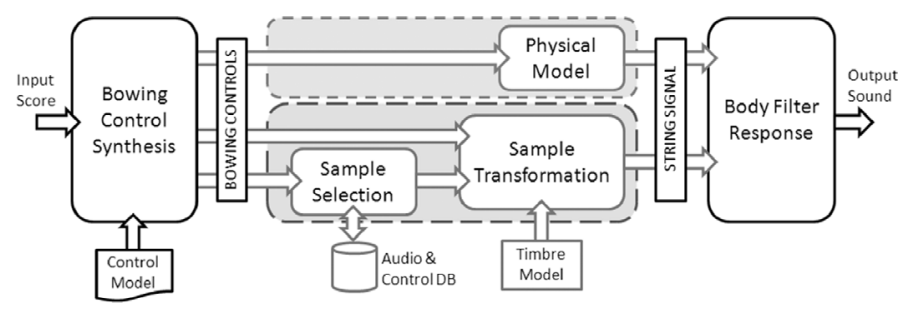

Fig. 2. Overview of the synthesis framework. Synthetic bowing controls are used both in physical modeling sound synthesis (top) and in sample-based sound synthesis (bottom).

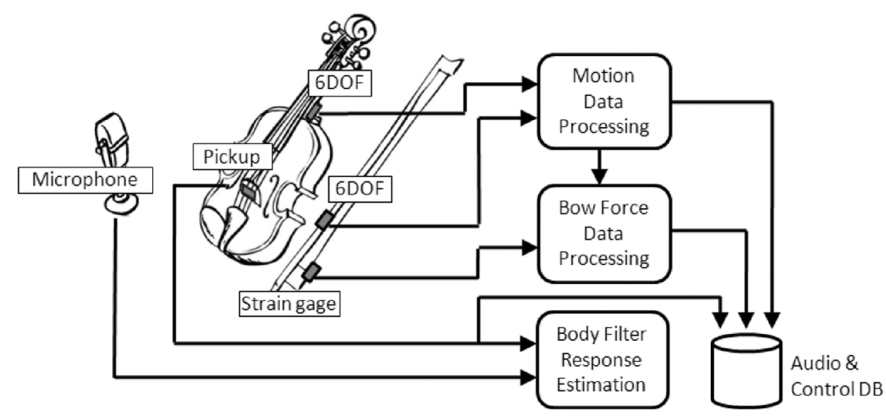

Fig. 3. Overview of the data acquisition setup. The database is constructed from processed motion, force, and audio data that has been automatically aligned to acquired audio and segmented at note-level.

transformation stages of a sample-based synthesizer, where the resulting sound signal (also corresponding to the string vibration) is also convolved with the estimated body impulse response. This work represents an extension of previous works [12], [28], [11], [29]-[32] put in the context of offline sound synthesis, providing a further advancement in the application of the current research results to sound generation.

\section{DAtA ACQUisition}

Both bowing control data and produced sound were acquired from real performance recordings in a reduced-intrusiveness sensor setup, including a microphone AKG 414,4 a bridge piezo-electric pickup Yamaha VNP1, 5 two six-degree of freedom (6DOF) sensors of the commercial tracking system Polhemus Liberty 6 each one providing position $(x, y, z)$ and rotation (azimuth, elevation, tilt), and a self-constructed device for sensing hair ribbon deformation based on strain gages.

An overview of the data acquisition is illustrated in Fig. 3. Bowing motion data was acquired by following the procedure introduced in [12], while bow force data was gathered using the techniques presented in [11], [9], and [30]. Both acquisition processes (bow motion and bow force) need a calibration process to be performed prior to recording sessions.

Recording scripts (including exercises and short musical pieces) were designed to cover four different articulation types (détaché, legato, staccato, and saltato), three different dynamics, and varied note durations in different performance contexts (attending to bow direction changes and silences). Score performance alignment was performed automatically by

\footnotetext{
${ }^{4}$ http://www.akg.com/

${ }^{5}$ http://www.yamaha.co.jp/english/product/strings/v_pickup/index.html

${ }^{6}$ http://www.polhemus.com/?page=Motion_Liberty
} 

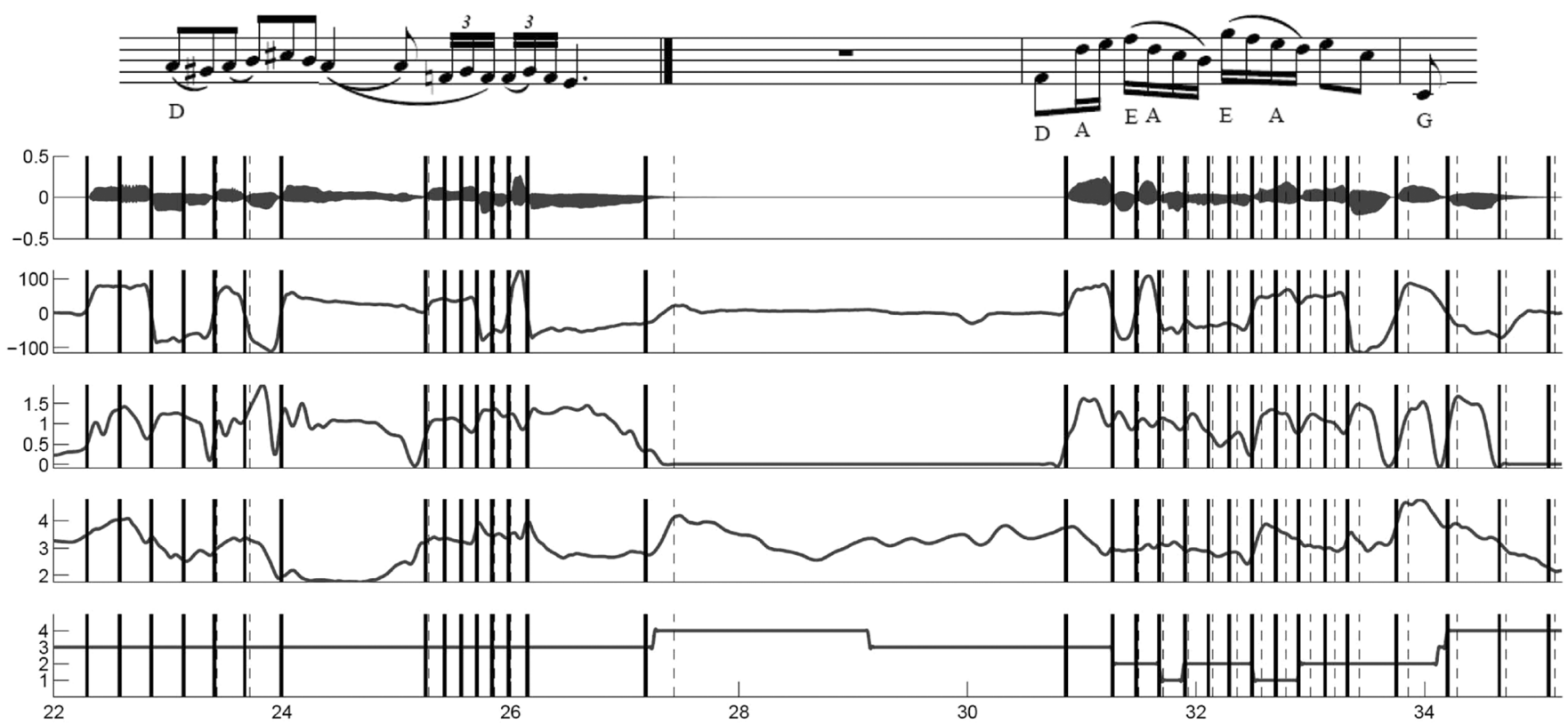

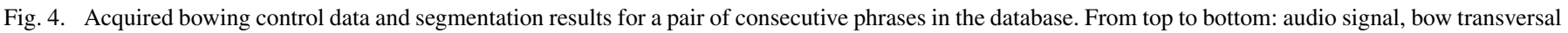

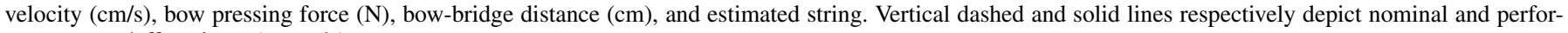
mance onset/offset times (seconds).

means of a dynamic programming adaptation (based on the Viterbi algorithm [33]) of the procedure introduced in [12]. The algorithm is based on both audio analysis data and on acquired bowing control parameters. When necessary, manual revision was performed as a postprocessing step in order to ensure the appropriate segmentation of bowing control and audio streams of approximately 11000 notes. Segmentation results for a pair of consecutive phrases from the database is shown in Fig. 4, where contours of relevant bowing control parameters are displayed.

An estimation of the body impulse response of the violin was carried out by deconvolving the string vibration signal (acquired with a bridge pickup) with the radiated sound signal (acquired with a microphone).

\section{A. Motion Data Processing}

Position and orientation of the two 6DOF sensors are tracked at $240 \mathrm{~Hz}$. The first is attached to the violin back plate, in the upper bout, at the neck edge. The second one is affixed to the bow wood, close to its center of gravity. From the data provided by these sensors, a set of motion descriptors is extracted by means of the data processing steps outlined in this section. For a more detailed description of the procedure for obtaining relevant bowing motion parameter streams, refer to [12].

Initially, a calibration of the string and hair ribbon ends is performed. The exact position of the eight string ends (four at the nut and four at the bridge) can be tracked by previously annotating (during calibration) their position relative to the coordinate system defined by the position and orientation of the 6DOF sensor placed in the violin. In a similar manner, the positions of the two hair ribbon ends (at the frog and at the tip) are estimated from the position and orientation of the 6DOF sensor placed on the bow. Both the violin plane and the bow plane (respectively

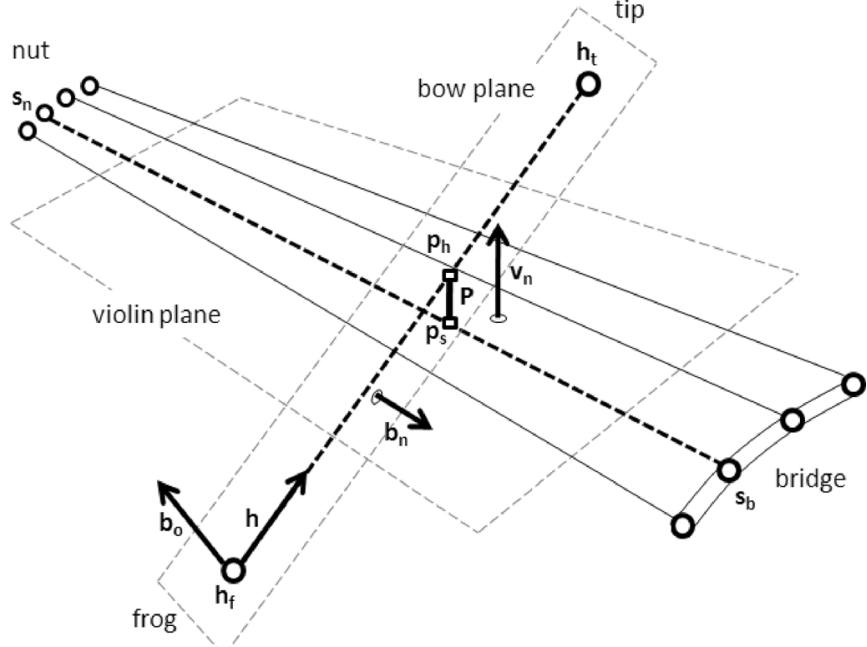

Fig. 5. Schematic representation of relevant positions and orientations tracked in the extraction of bowing motion parameters.

defined by their normal vectors $v_{n}$ and $b_{n}$ ) are estimated. The former is estimated from the eight string ends, and the latter is estimated from both the sensor position and from the two hair ribbon ends (see Fig. 5).

We estimate the bow tilt by measuring the angle between the violin plane normal vector $v_{n}$, and a vector $b_{o}$ being simultaneously parallel to the bow plane and perpendicular to the bow plane normal vector $b_{n}$ (the vector $b_{o}$ is obtained as the vectorial product of the hair ribbon vector $h$ and $b_{n}$ ). In a similar manner, the string being played is estimated by measuring the angle between $v_{n}$ and $h$ (see Fig. 5). By defining a line between the ends of the string being played (depicted as $s_{b}$ and $s_{n}$ ), and another line between the ends of the hair ribbon (depicted as $h_{f}$ and $h_{t}$ ), a segment $P$ is defined by a line perpendicular to both $p_{h}$ and 
$p_{s}$. The bow transversal position is defined as the distance between $p_{h}$ and $h_{f}$, and the bow-bridge distance is defined as the distance between $p_{s}$ and $s_{b}$. Bow velocity is obtained as the time derivative of the bow transversal position.

\section{B. Bow Force Data Processing}

Bow force is obtained using a dual strain gage device extending the ideas first proposed in [9] and later re-implemented in [11]. When playing on a string, the force applied by the performer is estimated from the deformation of the hair ribbon, to which a pair of strain gages are attached at the frog end. Each strain gage is attached to one side of a bending piece that is laying against the hair ribbon, thereby providing a measure of the hair ribbon deflection.

A calibration procedure was designed for obtaining real values of bow force from the voltage changes caused by the deformation of the gages under different bowing conditions (i.e., for different values of bow position and bow tilt). This calibration procedure, set as a prerecording step, entailed the construction of a bowing table on top of which a commercial force transducer was attached. The transducer holds a rolling cylinder (emulating the string) so that real values of pressing force can be acquired when bowing the cylinder. In addition, bow position and bow tilt were acquired by tracking bowing table orientation and position, in a similar manner as used for tracking the violin. Acquired values of bow force from the transducer were set as the target prediction in a support vector regression framework, with the inputs being the voltage readings coming from the strain gages, the acquired bow position, and acquired bow tilt.

Due to the long duration of the performance recordings used for constructing our database, hair ribbon tension changes happening during recording sessions caused inconsistencies in the calibration parameters. For tackling this problem, we redesigned the calibration procedure in order to make it incremental, so that hair ribbon tension changes are compensated (see [30] for a detailed description).

\section{Body Filter Response Estimation}

A body filter response (understood as the bridge pickup to radiated sound transfer function response) is estimated by means of deconvolving the acquired radiated sound signal (microphone) and the acquired string vibration signal (bridge pickup) [34], but in a frame-by-frame time-averaging fashion.

For doing so, we recorded a long duration glissando played on the $\mathrm{G}$ string (lower pitch) in order to capture low frequency excitation occurring during violin performance. For the acquisition of the radiated sound, we placed the microphone in front of the violin and asked the performer to keep the same position and orientation while playing the glissando, so that a low variability on distance and orientation minimized inconsistencies in our time-average bin-per-bin estimation.

The magnitude for each frequency bin is estimated as the average of individual frame estimations (one per frame), each one weighted by the energy of the frame. Conversely, the phase value for each bin is estimated as the maximum of a phase histogram that is constructed from individual phase estimations (one per frame), each one weighted by the energy of the frame.

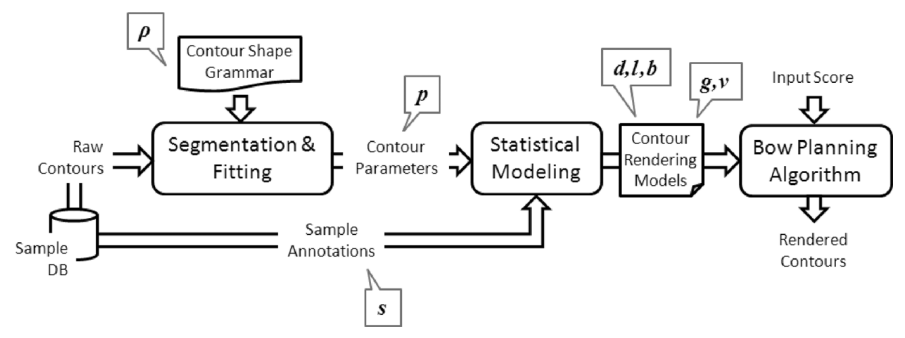

Fig. 6. Framework for analysis/synthesis of bowing parameter contours. Denomination letters given the relevant variables along the process are displayed.

\section{ANALYSIS OF BOWING PARAMETER CONTOURS}

We model the temporal contours of bow velocity, bow force, and bow-bridge distance as sequences of short segments, in particular Bézier cubic curve segments. Bow-bridge distance data are first transformed into a normalized parameter $\beta$ that is computed as the ratio between the bow-bridge distance and effective string length, having the effective string length defined as the distance between the bridge and the finger position. Considering different note articulations, dynamics, and contexts, a number of note classes is defined. Bowing parameter contours of the database corpus are analyzed at note-level by following a predefined grammar that dictates constraints of curve segment sequences for each of the classes in consideration. Based on dynamic programming, gesture parameter contour analysis provides a set of curve parameters for each note. Obtained curve parameters are sufficient for reconstructing original contours with significant fidelity, allowing the contours to be robustly represented in a relatively low dimensionality space. As illustrated in Fig. 6, contour representation parameters (e.g., curve parameters) are used for obtaining a rendering model able to generate contours of bowing control parameters given a set of note characteristics. The rendering model can be used within a bow planning algorithm for synthesizing bowing parameter contours from an input score.

In Fig. 6, we have displayed the denomination given to relevant variables used along the process of both analysis of contours (detailed in this section), and modeling of contours (introduced in Section IV). The grammar entries are denominated as $\rho$, and are needed for driving contour segmentation and fitting (see Section III-C). The curve parameter vectors obtained for each note sample by means of automatic segmentation and fitting are denominated as $p$ (see Section III-D). Performance context vectors (mainly derived from score annotations and used for performing clustering of note samples as detailed in Section IV-B) are denominated as $s$. The letters $d, l$, and $b$ are used for denominating the three one-dimensional normal distributions that are used to describe each of the duration clusters included in the model (see Section IV-C). Finally, each of the performance context sub-clusters also included in the model (see Section IV-B) is described by a curve parameter normal distribution $g$ and a performance context normal distribution $v$.

\section{A. Note Classification}

Concerning different score-annotation characteristics of the notes in the corpus, notes are divided into different classes for which specific bowing contour analysis is performed, and different models are later constructed. In order to set up the clas- 
sification basis, we attend first to intrinsic note characteristics (based on annotations attached to the note), leaving three categories: articulation, dynamics, and bow direction. In addition, two contextual characteristics (by looking to some characteristics of the surrounding notes) are considered: bow direction context and silence context. The possible labels for each characteristic are as follows:

\section{- Intrinsic characteristics}

[ART] Articulation type: \{détaché legato staccato saltato

[DY] Dynamics: \{piano mezzoforte forte $\}$

[BD] Bow direction: $\{$ downwards upwards $\}$

\section{- Contextual characteristics}

[BC] Bow context: $\{$ init mid end iso

[PC] Silence context: $\{$ init mid end iso $\}$

Considering intrinsic note characteristics, first and most important is the articulation type. We have considered four different articulations: détaché, legato, staccato, and saltato. Three different dynamics are present in the corpus: piano, mezzoforte, or forte. The possible bow directions are downwards and upwards.

In terms of what we refer to as contextual characteristics, we consider two main aspects: the position of a note within a bow (e.g., in legato articulation, several notes are played successively without any bow direction change), and the position of a note with respect to rest segments (e.g., silences). For the case of bow context, we classify a note as init when it is the first note played within a succession of notes sharing the same bow direction. A note is classified as mid when it is played neither first nor last. The class end corresponds to notes played last, while notes appearing as the only notes within a bow (e.g., in détaché articulation) are classified as iso. Similarly, the term silence context refers to successions of notes with no rest segments or silences in between. Respectively, notes will be classified as init when preceded by a silence and followed by another note, as mid when preceded and followed by a note, as end when preceded by a note and followed by a silence, and as iso when surrounded by silences.

Each possible combination of the five cited characteristics ${ }^{7}$ leads to a note gesture class $C_{\boldsymbol{i}}$. Collected bowing parameter streams for each class are treated separately in the contour analysis and synthesis parts. With enough recordings, more contextual variables could be taken into account such as the preceding and following articulations or metrical position.

\section{B. Contour Representation}

Bowing parameter contours of classified notes are modeled by sequences of a predefined number of parametric curve segments, in this case constrained cubic Bézier curves [21], [29], following a structured representation applied at note-level. We have represented the basic unit in Fig. 7 (left). The constraints in Fig. 7 (center) allow defining its shape by a vector $b=$ $\left[d v_{s} v_{e} r_{1} r_{2}\right]$, where $d$ represents the segment duration, $v_{s}$ represents the starting $y$-value, $v_{e}$ represents the ending $y$-value, and $r_{1}$ and $r_{2}$ represent the relative $x$-values of the attractors $p_{2}$ and $p_{3}$, respectively (possible shapes for extreme values of $r_{1}$ and $r_{2}$ are displayed in grey). We chose this as the building

\footnotetext{
${ }^{7}$ Note that not all of the combinations are feasible. For instance, detaché notes cannot share the bow direction with surrounding notes.
}
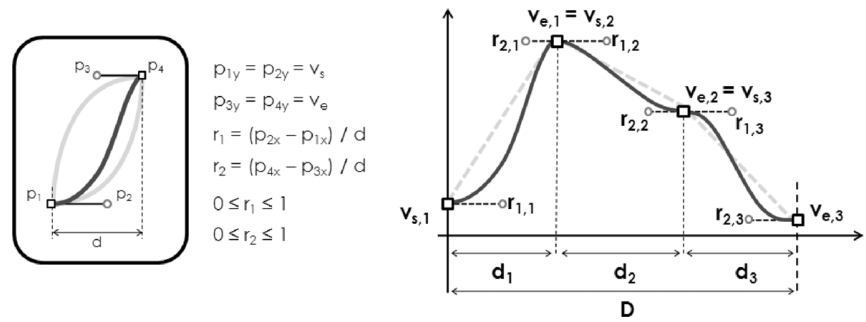

Fig. 7. Constrained Bézier cubic segment used as the basic unit in the representation of bowing parameter contours (left). Following the constraints given by the set of equations in the center, a sequence of segments can be defined for representing a particular shape (right), where the parameters needed for representing the contour are depicted along with the linear approximation of each of the segments.

block for modeling contours of bowing parameters because of its robustness and flexibility. Given a bowing parameter contour $q(t)$ with $t \in[0, d]$, its starting value $q(0)=v_{s}$, and its ending value $q(d)=v_{e}$, the optimal attractor relative $x$-values $r_{1}^{*}$ and $r_{2}^{*}$ leading to an optimal Bézier approximation $\sigma^{*}(t)$ of the segment can be found via constrained optimization [21].

\section{Grammar Definition}

Contours of bowing parameters (bow velocity, bow force, and $\beta$ ratio) of the samples belonging to each note class have been carefully observed in order to determine an optimal representation scheme when using sequences of constrained Bézier curve segments as previously introduced. While deciding on the scheme, the aim was to minimize the length of the sequences while preserving representation fidelity. For each of the note classes, we defined a grammar entry $\rho$ composed by three different tuples $\rho_{V}, \rho_{F}$, and $\rho_{\beta}$, each one corresponding to a bowing parameter (bow velocity, bow force, and bow-bridge distance). For a bowing parameter $b$, the tuple $\rho_{b}$ is defined by

$$
\rho_{b}=\left\{N_{b}, \Delta s_{b}^{*}\right\}
$$

where $N_{b}$ corresponds to the number of segments, and $\Delta s_{b}^{*}$ is a slope sequence constraint vector, both used during segmentation and fitting.

The slope constraint vector defines the expected sequence of slope changes for each bowing parameter contour. It can be written as

$$
\Delta s_{b}^{*}=\left[\delta s_{b, 1}^{*} \cdots \delta s_{b, N_{b}-1}^{*}\right]
$$

where each $\delta s_{i}^{*}$ is a slope change parameter defined for the $i$ th pair of successive segments. If each $i$ th segment is approximated linearly, a contour slope sequence $s=\left[s_{i} \cdots s_{N}\right]$ is obtained. Each of the $N-1$ pairs of successive slopes entail a slope change that can be either positive $\left(s_{i}<s_{i+1}\right)$ or negative $\left(s_{i}>s_{i+1}\right)$. In order to express an expectancy on the sequence of slope changes, a parameter $\delta s_{i}$ is defined for each of the $N-1$ pairs of successive segments. This parameter can take three different values: $\delta s_{i}^{*} \in\{-1,+1,0\}$. A value of $\delta s_{i}^{*}=0$ denotes no clear expectancy in the relationship between successive slopes $s_{i}$ and $s_{i+1}$. A value of $\delta s_{i}^{*}=1$ denotes expecting an increase in the slope value (i.e., $s_{i}<s_{i+1}$ ), while a value 
of $\delta s_{i}^{*}=-1$ denotes the opposite. This can be summarized as follows:

$$
\delta s_{i}^{*}= \begin{cases}0 & \text { if no expectancy on slope change } \\ 1 & \text { if expected } s_{i}<s_{i+1} \\ -1 & \text { if expected } s_{i}>s_{i+1}\end{cases}
$$

Observation of contours of the different classes led to the definition of a grammar entry for each note class. ${ }^{8}$ Analyzing other kinds of articulations or contexts (or even working with control parameter contours of other musical instruments) would of course lead to different grammar entries. An example tuple $\rho_{\text {ex }}$ of a grammar entry, defined for the hypothetic contour depicted in Fig. 7 (right), would be defined by $N_{\mathrm{ex}}=3$ and $\Delta s_{\mathrm{ex}}^{*}=\left[\begin{array}{ll}-1 & -1\end{array}\right]$.

\section{Automatic Segmentation and Fitting}

Segmentation and fitting are approached by automatically searching an optimal duration vector $d^{*}=\left[d_{1}^{*} \cdots d_{N}^{*}\right]$ such that a total approximation $\operatorname{cost} C$ is minimized while satisfying that the sum of all components of the duration vector must be equal to the note duration $D$. This is expressed in (4) and (5), where the approximation error $\xi_{i}$ for the $i$ th segment is computed as the mean squared error between the real contour $q_{i}(t)$ and its optimal Bézier approximation $\sigma_{i}^{*}(t)$ (see Section III-B), and $w_{i}$ corresponds to a weight applied to each $\xi_{i}$

$$
\begin{aligned}
d^{*} & =\left[d_{1}^{*} \cdots d_{N}^{*}\right]=\underset{d, \sum_{i=1}^{N} d_{i}=1}{\operatorname{argmin}} C(d) \\
C(d) & =\sum_{i=1}^{N-1} w_{i} \xi_{i}+\xi_{N}
\end{aligned}
$$

The weight $w_{i}$ applied to each of the first $N-1$ computed $\xi_{i}$ is set as penalty and depends on the fulfillment of the slope sequence constraints defined by $\Delta s^{*}$ (see Section III-C). For each pair of successive $i$ th and $(i+1)$ th segments derived from a candidate $d$, a parameter $\delta s_{i}$ is computed from the slopes $s_{i}$ and $s_{i+1}$ of their respective linear approximations as

$$
\delta s_{i}=\operatorname{sign}\left(s_{i+1}-s_{i}\right)
$$

The weight $w_{i}$ is set to an arbitrary value $W \gg 1$ in case $\delta s_{i}$ does not match its corresponding $\delta s_{i}^{*}$ in the grammar tuple, only when $\delta s_{i}^{*}$ was defined as nonzero. This can be expressed as

$$
w_{i}= \begin{cases}W \gg 1 & \text { if } \frac{\delta s_{i}}{\delta s_{i}^{*}}<1 \text { and } \delta s_{i}^{*} \neq 0, \\ 1 & \text { otherwise. }\end{cases}
$$

The solution for this problem is found by using dynamic programming [33]. From this analysis, the set of parameters defining the Bézier curve segments that best models each of the contours is obtained from each note in the corpus. Some examples of the results of automatic segmentation and fitting are shown in Fig. 8, where acquired bowing parameter contours are compared to their corresponding Bézier approximations for détaché, legato, staccato, and saltato articulations.

\footnotetext{
${ }^{8}$ http://www.dtic.upf.edu/ emaestre/gestureModels/bowed/violin/grammarV1.pdf
}

\section{Modeling of Bowing Parameter Contours}

From the results of automatic segmentation and fitting (see Section III-D), a curve parameter statistical model is obtained for each note class. First, curve parameter vectors (each represents the contours of the three bowing parameters in a note execution) are clustered by considering a set of performance context parameters obtained from annotations. Then, normal distributions of both curve parameters and performance context parameters are estimated from each cluster. A duration-specific statistical description is obtained for each of the clusters. The concept of this modeling approach is based on the notion that in a contour rendering context, statistical descriptions of bowing parameter contours can be retrieved from and adapted to an input performance context derived from the musical score.

\section{A. Contour Parameter Space}

The curve parameters of each note are represented as a vector $p$ resulting from the concatenation of three curve parameter vectors $p_{V}, p_{F}$, and $p_{\beta}$ (corresponding to the bow velocity, bow force, and $\beta$ ratio contours, respectively), and the bow displacement $\Delta \mathrm{BP}=\left|\mathrm{BP} \mathrm{OFF}-\mathrm{BP}_{\mathrm{ON}}\right|$ performed in the execution of the note. This is expressed as

$$
p=\left\{p_{V}, p_{F}, p_{\beta}, \Delta \mathrm{BP}\right\}
$$

Curve parameter vectors $p$ reside in a space whose dimensionality depends on the number of segments used for modeling bowing parameter contours (indeed defined by the corresponding grammar entry $\rho$, see Section III-C). Each of the three parameter vectors contains three different sub-vectors: a first sub-vector $p^{d}$ containing the relative durations $d_{i} / D$ of each of the segments, a second sub-vector $p^{v}$ containing the inter-segment $y$-axis values (starting or ending values $v_{s, i}$ or $v_{e, i}$ of each one of the segments), and a third sub-vector $p^{r}$ containing the pairs of attractor $x$-value ratios $r_{1, i}$ and $r_{2, i}$ [see Fig. 7 (right)]. Equations (9)-(12) summarize the organization of the parameters of a hypothetical parameter $b$, and is applicable to any of the three bowing parameters:

$$
\begin{aligned}
p_{b} & =\left\{p_{b}^{d}, p_{b}^{v}, p_{b}^{r}\right\} \\
p_{b}^{d} & =\left[d_{b, 1} / D \cdots d_{b, N_{b}} / D\right] \\
p_{b}^{v} & =\left[v_{b, s, 1} \cdots v_{b, s, N_{b}} v_{b, e, N_{b}}\right] \\
p_{b}^{r} & =\left[r_{b, 1,1} r_{b, 2,1} \cdots r_{b, 1, N_{b}} r_{b, 2, N_{b}}\right]
\end{aligned}
$$

\section{B. Performance Context-Based Sample Clustering}

Note samples of each note class are first separated according to the string played. Then, notes played on each string are clustered into different groups based on a set of performance context parameters, consisting in note duration $D$, starting bow position $\mathrm{BP}_{\mathrm{ON}}$ (distance from the frog to the string contact point), and string length $L_{\text {st }}$ (obtained from the fundamental frequency). In order to do so, each note is first annotated with a vector

$$
s=\left[D \mathrm{BP}_{\mathrm{ON}} L_{\mathrm{st}}\right]
$$




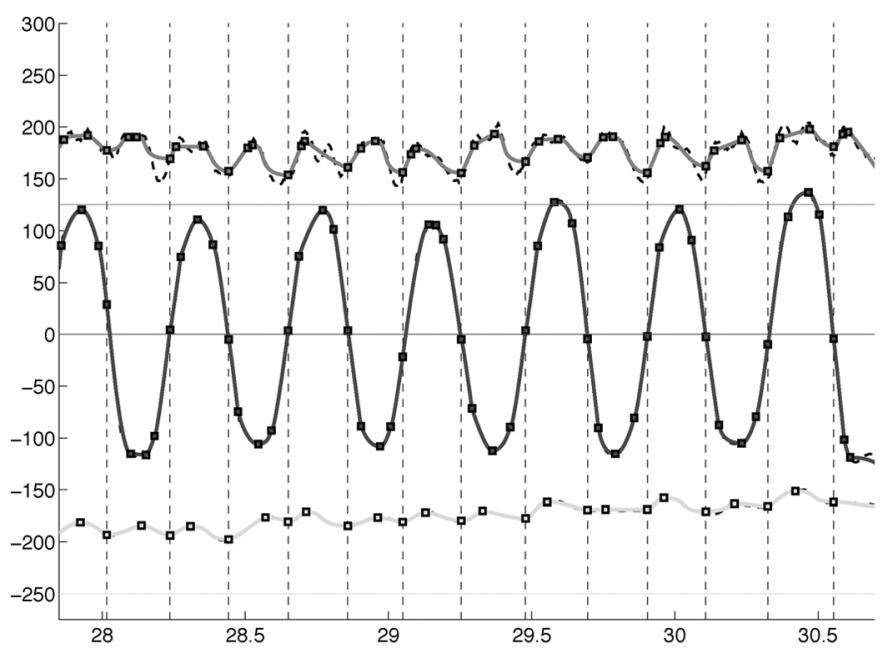

(a)

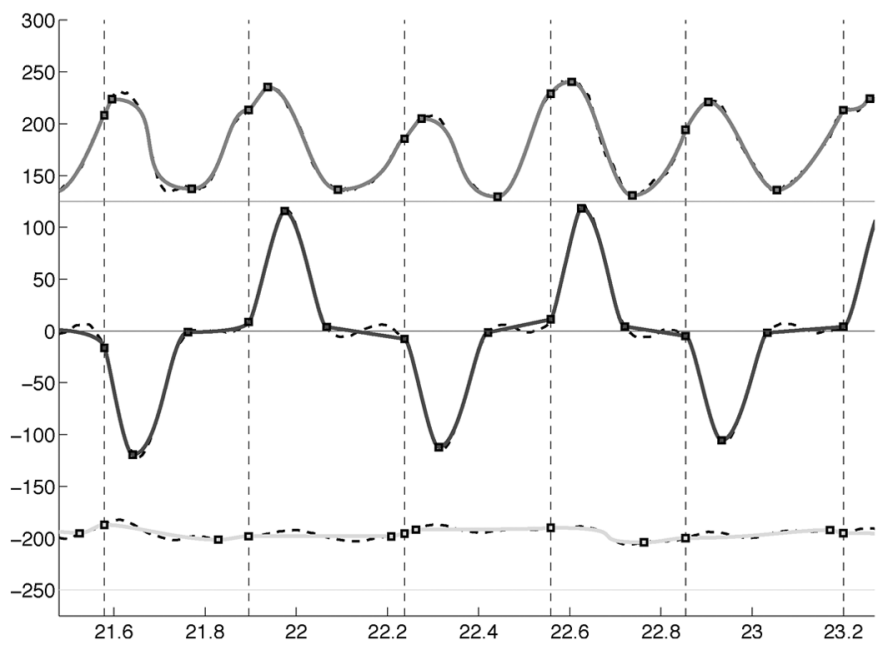

(c)

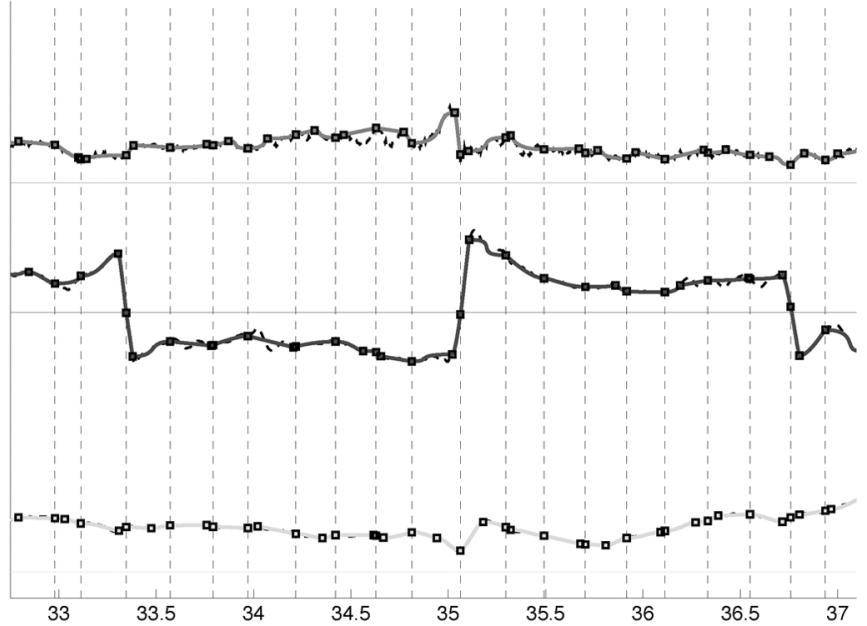

(b)

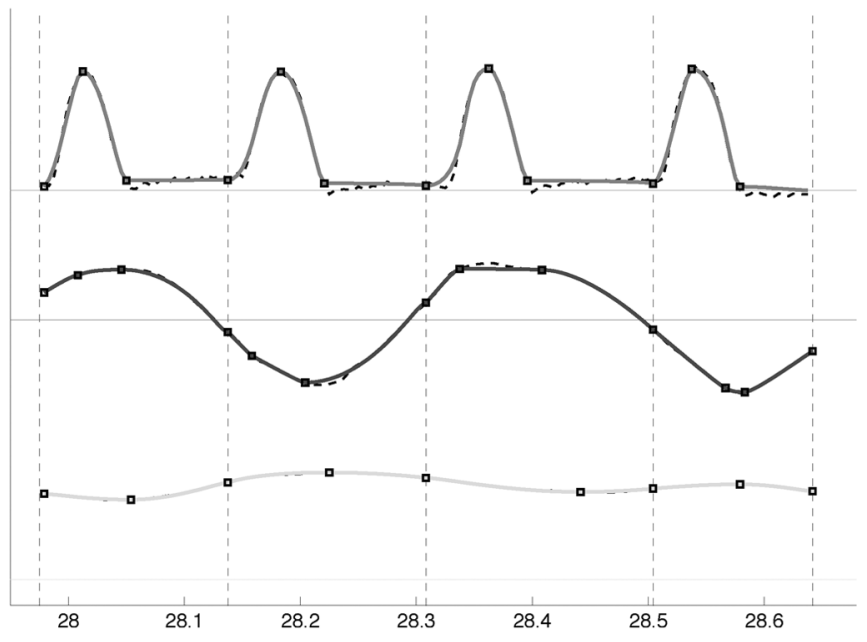

(d)

Fig. 8. Results of the automatic segmentation and fitting of bowing parameter contours. In each figure, from top to bottom: acquired bow force (0.02 N/unit), bow velocity $(\mathrm{cm} / \mathrm{s})$, and bow-bridge distance $(0.04 \mathrm{~cm} / \mathrm{unit})$ are depicted with thick dashed curves laying behind the modeled contours, represented by solid thick curves. Solid horizontal lines represent the respective zero levels. Junction points between successive Bézier segments are represented by black squares, while vertical dashed lines represent note onset/offset times (seconds). (a) Détaché articulation, (b) Legato articulation, (c) Staccato articulation, and (d) Saltato articulation.

and a two-step $k$-means clustering is performed. Due to considering note duration $D$ as the most important of the three context parameters in $s$, notes are clustered in two steps.

1) Duration-Based Clustering: In a first step, $N$ duration clusters of notes are obtained by applying the $k$-means clustering algorithm [35] to the note samples, based on the first component of the context vector $s$, i.e., the note duration $D$.

2) Performance Context-Based Clustering: In a second step, $M$ performance context sub-clusters of note samples are obtained by again applying the $k$-means clustering algorithm to the notes within each of the previously obtained $N$ duration clusters, but this time attending to the 3-D context vector $s$. Ideally, this leads to $N \times M$ performance context sub-clusters $c^{n, m}$ per note class $C_{i}$, each one containing a number of $p$ contour parameter vectors and $s$ performance context vectors (we denote these sets of vectors as $p^{n, m}$ and $s^{n, m}$ ), each pair corresponding to a note sample.

\section{Model Parameters Estimation}

The parameters defining a bowing parameter contour model of a note class consist of statistical descriptions of duration and bow displacement attributes for each of the $N$ duration clusters, as well as statistical descriptions of curve parameter vectors and performance context attributes for each of the $M$ performance context sub-clusters within each of the duration clusters.

1) Duration Cluster Description: Initially, a note duration normal distribution

$$
d^{n}=\left\{\tau^{n}, \Phi^{n}\right\}
$$

defined by a mean duration $\tau^{n}$ and a duration variance $\Phi^{n}$ is estimated from the duration $D$ of the notes contained in the $n$th duration cluster.

Secondly, for each duration cluster and each bowing parameter $b$, an analysis is performed on the correlation between the 
absolute durations $\left[d_{b, 1} \cdots d_{b, N_{b}}\right]$ of the $N_{b}$ Bézier segments and the note duration $D$. We include such information in the model in order to be able to adequately adjust segment relative durations when reconstructing contours (see Section V). The task of this analysis is to find which of the $N_{b}$ segments presents the highest correlation with the note duration $D$. In order to do so, we first collect all note samples belonging to the duration cluster under analysis, and compute the Pearson correlation coefficient $r\left(d_{b, i}, D\right)$ between each segment absolute durations $d_{b, i}$ of the $N_{b}$ segments, and the note duration $D$. Then, we select the segment $l^{n, b}$ presenting the highest correlation:

$$
l^{n, b}=\underset{i, i=1 \cdots N_{b}}{\operatorname{argmax}} r\left(d_{b, i}, D\right) .
$$

As a result, a duration correlation vector

$$
l^{n}=\left\{l^{n, V}, l^{n, F}, l^{n, \beta}\right\}
$$

containing obtained segment numbers $l^{n, V}, l^{n, F}, l^{n, \beta}$ for each of the three contours, is attached to each $n$th duration cluster.

Finally, a bow displacement distribution

$$
b^{n}=\left\{\pi^{n}, \Pi^{n}\right\}
$$

defined by a mean bow displacement $\pi^{n}$ and a bow displacement variance $\Pi^{n}$, is estimated from the bow displacement $\Delta \mathrm{BP}$ annotated for each note within the notes of the $n$th duration cluster.

2) Performance Context Sub-Cluster Description: Assuming that both the curve parameter vectors $p^{n, m}$ and the context vectors $s^{n, m}$ follow a normal distribution, we estimate $N \times M$ pairs of normal distributions $g^{n, m}$ and $v^{n, m}$. The distribution $g^{n, m}$ is estimated from the set $p^{n, m}$ of curve parameter vectors contained in the cluster $c^{n, m}$, and is defined by a mean vector $\mu^{n, m}$ and covariance matrix $\Sigma^{n, m}$. Analogously, the distribution $v^{n, m}$ is estimated from the set $s^{n, m}$ of performance context vectors contained in the cluster $c^{n, m}$, and is defined by a mean vector $\gamma^{n, m}$ and covariance matrix $\Omega^{n, m}$. The two normal distributions describing each sub-cluster can expressed as follows:

$$
\begin{aligned}
& g^{n, m}=\left\{\mu^{n, m}, \Sigma^{n, m}\right\} \\
& v^{n, m}=\left\{\gamma^{n, m}, \Omega^{n, m}\right\} .
\end{aligned}
$$

\section{Model Parameters Overview}

Thus, the set of parameters describing the model for each note class $C_{i}$ will contain the following.

- $N$ duration clusters, each one defined by:

- a duration normal distribution $d^{n}$;

- a segment duration correlation vector $l^{n}$;

- a bow displacement normal distribution $b^{n}$.

- $M$ performance context sub-clusters within each of the $N$ duration clusters. Each of the $N \times M$ performance context sub-clusters is defined by:

- A performance context normal distribution $v^{n, m}$;

- A curve parameter normal distribution $g^{n, m}$.

\section{E. Discussion}

In our implementation, the clustering procedure was set up as an iterative process, having the initial parameters to be $N=3$ (three duration clusters) and $M=9$ (nine performance context sub-clusters). As a result of design principles followed in the creation of the set of exercises used as recording scripts, a sufficient number of notes of at least three different durations was ensured for almost every combination of articulation type, bow direction, and dynamics. Whenever very few note samples were found in any of the $M$ performance clusters, $M$ was reduced and the second-step clustering was repeated, yielding to values of $3 \leq M \leq 9$. For the specific case of staccato and saltato articulations, some of the note classes are better represented by just two duration clusters $(N=2)$. Clustering parameters (and hence the modeling capability of the clusters) is of course dependent on the size and nature of the database.

The justification of the decision not to consider the bow displacement $\triangle \mathrm{BP}$ as a variable for clustering is important to mention. The definition of the variables constituting the performance context parameter space is strongly linked to data availability. Comparing histograms of bow displacement to histograms of note duration revealed these two variables to be considerably correlated, and it was difficult to cluster bow displacement values in different groups. Converse to this behavior is that observed when comparing duration to effective string length or bow starting position, for which distributions appeared more flat, which led to this decision. With an optimal database coverage, an explicit introduction of the bow displacement as one of the performance context parameters could have been possible.

Notwithstanding the explanations given in the above paragraph, the bow displacement is indeed taken into account during the contour rendering stage by attending to the cross-correlation between the different curve parameters and the bow displacement itself (remember that the bow displacement has been included as an extra dimension when constructing the contour parameter vectors). When a contour parameter vector is generated from the resulting mixed Gaussian distribution, a bow displacement value comes along [it is one of the dimensions, see (8)]. It is likely that such a value of bow displacement does not match the target value provided by the bow planning algorithm, which implies that the bow displacement resulting from the generated contour of bow velocity will likely differ from the target. The intention is to meaningfully adapt the contour parameters (including those defining the bow velocity contour) so that the bow displacement (and any other that is required, for example in contour concatenation) is matched while respecting the cross-correlation between variables. The process, based on least-squares, is detailed in Section V-C.

\section{Synthesis of Bowing PARAMETER Contours}

By using the model parameters, curve parameter values corresponding to each bowing parameter contour are obtained for a given note present in an annotated input score. First, the note class to which each note belongs is determined following the principles outlined in Section III-A and detailed in [29]. Then, a target context performance vector $s^{t}=\left[D^{t} \mathrm{BP}_{\mathrm{on}}^{t} L_{\mathrm{st}}^{t}\right]$ (see Section IV-B) is determined from the score annotations. Based on $s^{t}$, a mixed curve parameter normal distribution $g^{*}$ is obtained from the contour parameter normal distributions $g$ contained in the model of the class in consideration. From $g^{*}$, a 
curve parameter vector $p$ [see (8)] can be drawn. Although obtained vectors $p$ will preserve the original variance, a number of constraints must be fulfilled by some curve parameters present in $p$, so some components of $p$ must be tuned before $p$ can be used for rendering the bowing parameters of the given note.

\section{A. Contour Parameters Distribution Mixing}

In this section, we detail the steps followed for obtaining the mixed curve parameter distribution $g^{*}$ based on a target performance context vector $s^{t}$. The vector $s^{t}$ is determined by the target note duration $D^{t}$ (duration in the input score), effective string length $L_{\mathrm{st}}^{t}$ (obtained from the scripted string and the pitch of the note), and starting bow position $\mathrm{BP}_{\mathrm{ON}}^{t}$. In the event that the process for synthesizing bowing parameter contours of a note is used as part of the bow planning algorithm introduced in Section VI-A, $\mathrm{BP}_{\mathrm{ON}}^{t}$ is estimated at each of the steps of the algorithm. Conversely, if contours are to be synthesized without using the bow planning algorithm, $\mathrm{BP}_{\mathrm{ON}}^{t}$ is set to the mean $\pi^{n^{*}}$ of the selected duration cluster (see Section IV-C1).

1) Duration Cluster Selection: First, the appropriate duration cluster $n^{*}$ (see Section IV-B) is selected. In doing so, we compute the normalized Euclidean distance between the target duration $D^{t}$ and each of the $N$ cluster duration distributions $d^{n}$ as

$$
n^{*}=\underset{n}{\operatorname{argmin}} \sqrt{\frac{\left(D^{t}-\tau^{n}\right)^{2}}{\left(\Phi^{n}\right)^{2}}} .
$$

2) Performance Context Cluster Selection: Within the selected duration cluster $n^{*}$, the closest $K$ performance context clusters (see Section IV.B) to the target context vector $s^{t}$ are selected from the $M$ sub-clusters in cluster $n^{*}$. The selection is performed by measuring the Mahalanobis distance

$$
D_{M}\left(s^{t}, v^{n^{*}, m}\right)=\sqrt{\left(s^{t}-\gamma^{n^{*}, m}\right)^{T}\left(\Omega^{n^{*}, m}\right)^{-1}\left(s^{t}-\gamma^{n^{*}, m}\right)}
$$

between $s^{t}$ and each $m$ th context vector distribution $v^{n^{*}, m}$ in $n^{*}$, and keeping a vector $h$ of length $K$ with the indexes of the performance context clusters in increasing order.

3) Curve Parameter Distribution Mixing: The mixed curve parameter distribution

$$
g^{*}=\left\{\mu^{*}, \Sigma^{*}\right\}
$$

from which we will draw a synthetic curve parameter vector $p$, is obtained as a weighted average of the $K$ source curve parameter distributions corresponding to the closest $K$ performance context distributions to the performance target $s^{t}$. The mixed curve parameter distribution parameters $\mu^{*}$ and $\Sigma^{*}$, respectively, correspond to the weighted average of the means and covariance matrices of the $K$ source curve parameter distributions. This can be written as

$$
\mu^{*}=\sum_{i=1}^{K} w_{i} \mu^{n^{*}, h(i)}
$$

$$
\Sigma^{*}=\sum_{i=1}^{K} w_{i} \Sigma^{n^{*}, h(i)} .
$$

For the weights $w_{i}$ corresponding to each distribution in the mix, we have used the Mahalanobis distances computed in the previous step, yielding

$$
w_{i}=\frac{D_{M}^{-1}\left(s^{t}, v^{n^{*}, h(i)}\right)}{\sum_{k=1}^{K} D_{M}^{-1}\left(s^{t}, v^{n^{*}, h(k)}\right)} .
$$

Note that choosing a good value for $K$ is very dependent on how data are distributed. It is more convenient to keep a low value for $K$, so that averaging of original distributions $g$ does not overly reduce the variance of the curve parameters in the resulting $g^{*}$.

\section{B. Contour Parameters Tuning}

After drawing an initial curve parameter vector $p$ from the mixed distribution $g^{*}$, its components are checked for the satisfaction of a set of constraints, some dealing with the nature of the curve segment model (for instance, attractor relative durations must be greater than zero), others dealing with the nature of the note class (e.g., related to articulation or bow/silence contexts), and others dealing with the relative duration of segments, or with note concatenation. Also, due to the nature of the model (some dimensions do not exactly follow a normal distribution), some values of the curve parameters in generated $p$ might not respect some of the aforementioned constraints. As already mentioned in Section IV-E, the bow displacement is one of the parameters to be adjusted. Many other small adjustments are performed in the actual implementation, but here we will just outline the adjustment of the components of $p$ involved in the relative durations of the segments, and in note contour concatenation issues. At the end of the section, we introduce a methodology devised for preserving the original likelihood of generated $p$ while satisfying any of these constrains. The methodology, based on least-squares, ensures that the probability of a new $p_{f}$ (after applying the adjustments) is maintained by performing required extra changes to those variables not subject to any constraint. More details can be found in [32].

1) Segment Relative Durations: The relative segment durations $d_{i}$ must sum to unity for each of the three contours. In order to perform the adjustments, the segment duration that is given by $l^{n^{*}, b}$ (which corresponds to the one found presenting the highest correlation with the note duration, see Section IV-C) is modified. This applies to any of the three bowing parameters. In the contour parameter vector $p$, the value of the relative duration $d_{b, l^{n^{*}, b}} / D$ (indeed corresponding to the segment $l^{n^{*}, b}$ of the bowing parameter $b$ ) is set to a value that, given the other relative durations, makes the relative durations sum to unity. This can be expressed as

$$
d_{b, l^{n^{*}, b}} / D=1-\sum_{\substack{i=1 \\ i \neq l^{n *}, b}}^{N_{b}} d_{b, i} / D
$$


where $D$ corresponds to the note target duration, and $N_{b}$ corresponds to the number of segments used for modeling the contour of the bowing parameter $b$.

2) Note Concatenation: Possible discontinuities of bowing parameter contours of successive notes are solved as follows. The starting value of the first segment of the sequence $\left(v_{s, 1}\right)$ of each $i$ th note is set to the ending value of the $N$ th segment (i.e., the last segment) of the sequence $\left(v_{e, N}\right)$ obtained for its predecessor $(i-1)$ th note (see Fig. 7). This applies to any of the three bowing parameter contours (bow velocity, bow force, and $\beta$ ratio), and gets naturally integrated in the bow planning algorithm presented in the next Section.

\section{Tuning the Contour Parameter Vectors While Preserving Likelihood}

Let $g^{*}$ be the mixed normal distribution describing the model curve parameters for a given note class and a target performance context (see Section V-A), and let $Q$ be the dimensionality of vectors in the space where the distribution $g^{*}$ is defined. Once an initial sample curve parameter vector $p_{i} \in \mathbb{R}^{Q}$ is drawn from $g^{*}$, a subset of $R$ parameters (with $R<Q$ ) contained in vector $p_{i}$ must be tuned by adding a constraint vector $\delta p_{i} \in \mathbb{R}^{Q}$ of the form $\delta p_{i}=\left[\delta p_{i_{1}}, \ldots, \delta p_{i_{R}}, 0, \ldots, 0\right]^{T}$ in order to satisfy a set of constraints (see above). In the event that the likelihood of the resulting parameter vector $p_{f}$ has decreased with respect to the initial $p_{i}$, the non-fixed $Q-R$ parameters of vector $p_{i}$ will be modified by adding a vector $\delta p_{f} \in \mathbb{R}^{Q}$ of the form $\delta p_{f}=$ $\left[0, \ldots, 0, \delta p_{f_{R+1}}, \ldots, \delta p_{f_{Q}}\right]^{T}$, so that the squared Mahalanobis distance $D^{2}{ }_{M}\left(p_{f}, p_{i}\right)$ between the initial and final vectors $p_{i}$ and $p_{f}$ is minimized.

Expressing $\delta p_{f}$ as the product of a $Q \times(Q-R)$ selector matrix $A$ and a vector $\delta p_{f_{0}} \in \mathbb{R}^{Q-R}$ of the form $\delta p_{f_{0}}=$ $\left[\delta p_{f_{01}}, \ldots, \delta p_{f_{0 Q-R}}\right]^{T}$, we write $p_{f}$ and $A$ as

$$
\begin{gathered}
p_{f}=p_{i}+\delta p_{i}+A \delta p_{f_{0}} \\
A=\left[\begin{array}{ccccc}
0 & 0 & \cdots & 0 & 0 \\
\vdots & \vdots & \ddots & \vdots & \vdots \\
0 & 0 & \cdots & 0 & 0 \\
1 & 0 & \cdots & 0 & 0 \\
0 & 1 & \cdots & 0 & 0 \\
\vdots & \vdots & \ddots & \vdots & \vdots \\
0 & 0 & \cdots & 1 & 0 \\
0 & 0 & \cdots & 0 & 1
\end{array}\right] .
\end{gathered}
$$

The problem is to obtain the final sample parameter vector $p_{f}$ by means of finding the optimal $\delta p_{f_{0}}{ }^{*}$. With the initial sample parameter vector $p_{i}$ fixed, the constraint vector $\delta p_{i}$, and the selector matrix $A$, we can express the search of $\delta p_{f_{0}}{ }^{*}$ as

$$
\begin{aligned}
\delta p_{f_{0}}{ }^{*} & =\underset{\delta p_{f_{0}}}{\operatorname{argmin}} f\left(\delta p_{f_{0}}\right) \\
& =\underset{\delta p_{f_{0}}}{\operatorname{argmin}} D^{2}{ }_{M}\left(p_{f}, p_{i}\right) \\
& =\underset{\delta p_{f_{0}}}{\operatorname{argmin}}\left(p_{f}-p_{i}\right)^{T} \Sigma^{*-1}\left(p_{f}-p_{i}\right)
\end{aligned}
$$

where $\Sigma^{*}$ represents the covariance matrix of $g^{*}$. By using (27), we can rewrite (29) as

$$
\delta p_{f_{0}}{ }^{*}=\underset{\delta p_{f_{0}}}{\operatorname{argmin}}\left(\delta p_{i}+A \delta p_{f_{0}}\right)^{T} \Sigma^{*-1}\left(\delta p_{i}+A \delta p_{f_{0}}\right) .
$$

In order to solve the problem, we make the gradient $\nabla_{\delta p_{f_{0}}} f\left(p_{f_{0}}\right)$ equal to zero, yielding

$$
\nabla_{\delta p_{f_{0}}} f\left(\delta p_{f_{0}}\right)=2 \delta p_{i}^{T} \Sigma^{*-1} A+2 A^{T} \Sigma^{*-1} A \delta p_{f_{0}}=0 .
$$

By solving for $\delta p_{f_{0}}$, we obtain the solution for $\delta p_{f_{0}}{ }^{*}$ as

$$
\delta p_{f_{0}}{ }^{*}=-\left(A^{T} \Sigma^{*-1} A\right)^{-1} A^{T} \Sigma^{*-1} \delta p_{i} .
$$

\section{Bow PlanNing AlgORITHM}

For a note to be executed in the context of a note sequence found in a score, the starting bow position and the bow displacement are chosen by the performer, who is able to plan the sequence of starting bow positions and bow displacements based on the constraints imposed by the finite length of the bow and on his/her preferences. In order to represent possible particularities in note executions given the different bow starting position and bow displacement possibilities, we have devised a bow planning algorithm able to take those into account. The two additional variables $\mathrm{BP}_{\mathrm{ON}}$ and $\triangle \mathrm{BP}$ introduced in the model (needed for obtaining curve parameters from a mixed distribution $g^{*}$ ) come into play, where the search for their appropriate values partially drive the individual rendering of bowing parameter contours of each note in the input score.

\section{A. Algorithm Description}

We set up the bow-planning problem as the task of finding an optimal sequence of note bow starting and ending positions, represented by the vector

$$
\mathrm{BP}^{*}=\left[\mathrm{BP}_{\mathrm{ON}, 1}{ }^{*} \mathrm{BP}_{\mathrm{OFF}, 1} * \cdots \mathrm{BP}_{\mathrm{ON}, N}{ }^{*} \mathrm{BP}_{\mathrm{OFF}, N}{ }^{*}\right]
$$

in which the starting bow position of each note matches the ending bow position of the previous note in the sequence:

$$
\mathrm{BP}_{\mathrm{ON}, n}=\mathrm{BP}_{\mathrm{OFF}, n-1} \quad \forall n=2, \ldots, N .
$$

For doing so, we set up the state transition matrix represented in Fig. 9, for which the number of columns is fixed by the number of notes in the sequence, and the number of rows is arbitrarily set by the desired resolution used for representing the bow position $\mathrm{BP} \in\left[0, L_{B}\right]$, with the string length $L_{B}$ being equal to $63 \mathrm{~cm}$.

In Fig. 9, each $n$th note is represented by an arrow going 1) from its onset to its offset in the x-axis (columns), and 2) from the starting bow position $\mathrm{BP}_{\mathrm{ON}}^{n}$ to the ending bow position $\mathrm{BP}_{\mathrm{OFF}}^{n}$ in the $y$-axis (rows). All possible executions of a note (associated to possible combinations of starting and ending bow positions) are assigned an execution cost $C_{n}$ (see Section VI-B). 


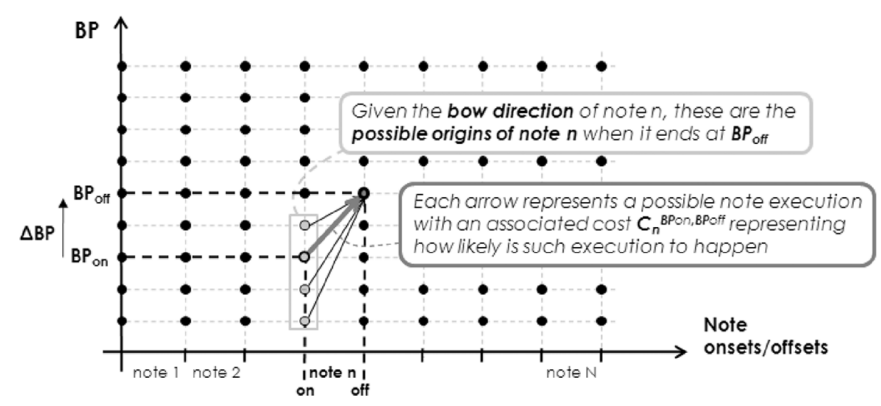

Fig. 9. Schematic illustration of the state transition matrix on which the bow planning algorithm is based. For each note in the input sequence, all possible combinations of starting and ending bow positions $\mathrm{BP}_{\mathrm{ON}}$ and $\mathrm{BP}$ OFF are assigned an execution cost $C$.

The optimal path, represented by the vector $\mathrm{BP}^{*}$, is found as the path minimizing the total cost $\Psi(\mathrm{BP})$

$$
\mathrm{BP}^{*}=\underset{\mathrm{BP}}{\operatorname{argmin}} \Psi(\mathrm{BP})
$$

having the total cost $\Psi(\mathrm{BP})$ defined as the sum of the execution costs of the notes

$$
\Psi(\mathrm{BP})=\sum_{n=1}^{N} C_{n}^{\mathrm{BP}_{\mathrm{ON}}^{n}, \mathrm{BP}_{\mathrm{OFF}}^{n}} .
$$

For each note in the sequence, the first step is to assign it a note class. Then, scripted note duration $D^{t}$ and the effective string length $L_{\mathrm{st}}^{t}$ (the latter computed from the scripted pitch and string) are obtained from the input score. In order to complete the performance context target vector $s^{t}$, the starting bow position $\mathrm{BP}_{\mathrm{ON}}^{t}$ and a bow displacement $\Delta \mathrm{BP}$ are needed. These two additional values are defined for each possible execution of the note, i.e., each possible combination of $\left\{\mathrm{BP}_{\mathrm{ON}},\right\}$ with $\mathrm{BP}_{\mathrm{ON}}, \mathrm{BP}_{\mathrm{OFF}} \in[0,63]$. While $\mathrm{BP}_{\mathrm{ON}}^{t}$ is used during distribution mixing (see Section $\mathrm{V}-\mathrm{A} 3$ ), the value of $\triangle \mathrm{BP}$ is set as one of the constraints for tuning curve parameters (see Section $\mathrm{V}-\mathrm{C}$ ).

In the algorithm, any value of $\mathrm{BP}_{\mathrm{OFF}}$ is allowed for every note, and the bow direction of the note will define the possible values of $\mathrm{BP}_{\mathrm{ON}}$, as it is expressed in

$$
\mathrm{BP}_{\mathrm{ON}} \in \begin{cases}{\left[0, \mathrm{BP}_{\mathrm{OFF}}\right)} & \text { if downwards bow direction } \\ \left(\mathrm{BP}_{\mathrm{OFF}}, L_{B}\right] & \text { if upwards bow direction. }\end{cases}
$$

The solution for $\mathrm{BP}^{*}$ is found by using dynamic programming techniques [33]. In our implementation, relatively short rests or silences are considered as if they were normal notes, with the aim of representing the bow traveling distance constraints associated to them. For that, they are treated as short détaché notes, using a détaché articulation model with duration-dependent dynamics for emulating different bow velocities. When necessary, any given input score might be divided into several sequences of notes depending on the length of the rests or silences found.

\section{B. Cost Computation}

Once the tuned curve parameter vector $p_{f}$ has been obtained from $g^{*}$ (see Sections VI-A and V) for a candidate execution, its associated cost $C$ is computed as follows. Given the set of $K$ source distributions $g^{n *, h(i)}$ used for obtaining the mixed distribution $g^{*}$ (see Section $\mathrm{V}-\mathrm{A}$ ), the cost $C$ is computed as a weighted sum of $K$ negative log-likelihoods of the vector $p_{f}$, each one computed given the corresponding $i$ th original distribution. This is expressed as

$$
C=\eta+\sum_{i=1}^{K} \omega_{i} n \log L\left(p_{f} \mid g^{n *, h(i)}\right)
$$

where $\omega_{i}$ represents the weight applied to each of the likelihoods, and $\eta$ is an additional cost related to the bow displacement $\triangle \mathrm{BP}$ of the current execution candidate.

The value of $\omega_{i}$ used for weighting each $i$ th likelihood is set to the Mahalanobis distance from the target performance context point $s^{t}=\left[D^{t} \mathrm{BP}_{\mathrm{on}}^{t} L_{\mathrm{st}}^{t}\right]$ to the centroid $\gamma^{n^{*}, h(i)}$ of the $i$ th source distribution $v^{n^{*}, h(i)}$ (see Section V-A), computed as in (25).

In order to further penalize note executions for which the bow displacement $\triangle \mathrm{BP}$ (determined by the candidate execution) is not likely to happen, we introduce the weight $\eta$. Since we have not pursued an in-depth study of performer preferences when choosing the bow displacement, we chose to pay attention to the bow displacement distribution $b^{n^{*}}$ associated with the selected duration cluster $n^{*}$ from the model being used (see Section IV-C). The penalty $\eta$ is computed as

$$
\eta=n \log L\left(\Delta B P \mid b^{n^{*}}\right) .
$$

\section{Gesture Parameter Contour Concatenation}

Gesture contour parameters of successive notes are naturally concatenated within the bow planning framework by taking advantage of the partial optimal path search that characterizes the Viterbi algorithm [33]. For a given $i$ th note and a given ending bow position $\mathrm{BP} \mathrm{OFF}_{i}$, the curve parameter vectors $p$ (each associated to a candidate $\mathrm{BP}_{\mathrm{ON}, i}$ and used for computing its associated execution cost, see Section VI-B) are known when computing the cost associated to the $(l+1)$ th note. For those candidate executions of the $(i+1)$ th note that have their starting bow positions $\mathrm{BP}_{\mathrm{ON}, i+1}$ matching $\mathrm{BP}_{\mathrm{OFF}, i}$, the starting values of the three contours of the $i+1$ th note will be set to the ending values obtained for the $i$ th note ending at $\mathrm{BP}_{\mathrm{OFF}, i}$. Setting these values is considered as a contour parameter constraint to be adjusted before computing the cost associated with the note (see Section V-C).

\section{SOUND SYNTHESIS}

Along previous chapters, we introduced a systematic approach to the acquisition, analysis, modeling, and synthesis of bowing controls in violin classical performance. The framework, which could be seen as an analysis/synthesis platform for instrumental gestures, is applied to generating effective bowing controls from an input score. The next subsections describe how such bowing controls are used as a key element for realistic violin sound generation, both in physical models and in sample-based synthesizers.

\section{A. Parameter Control Rendering}

Some results of bowing parameter contour rendering, obtained through an implementation of the modeling approach 


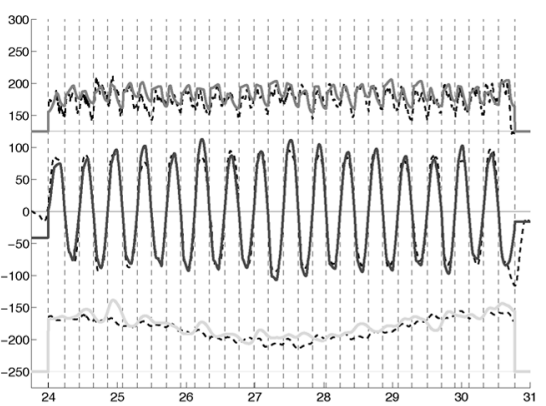

(a)

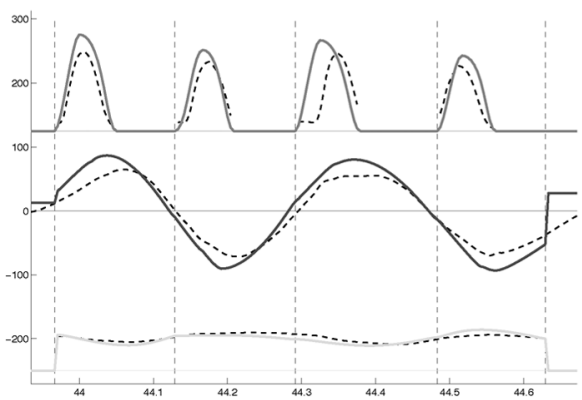

(d)

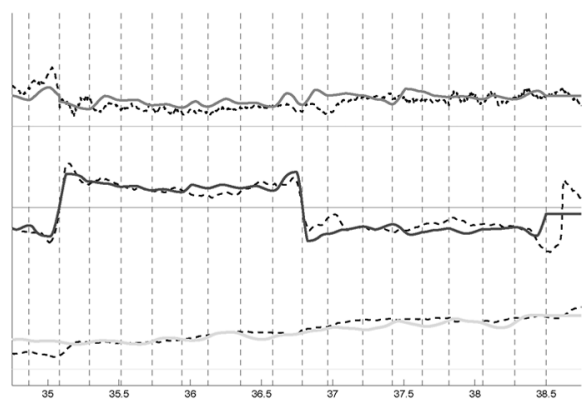

(b)

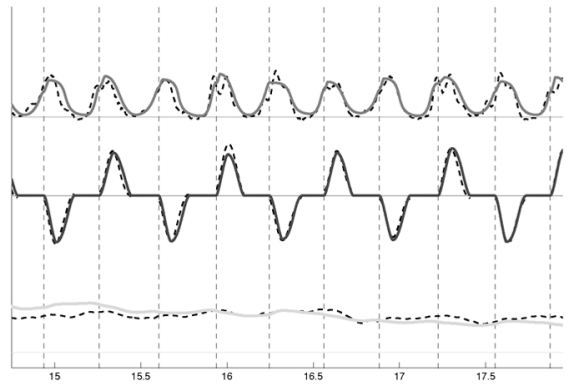

(e)

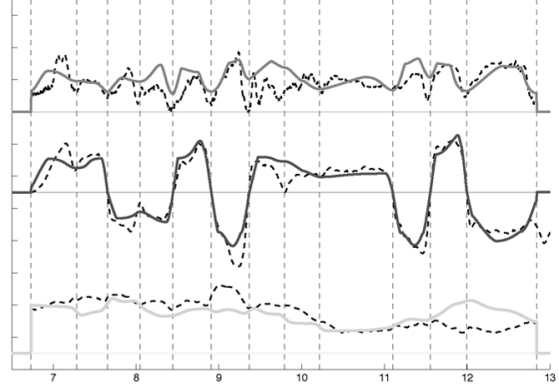

(c)

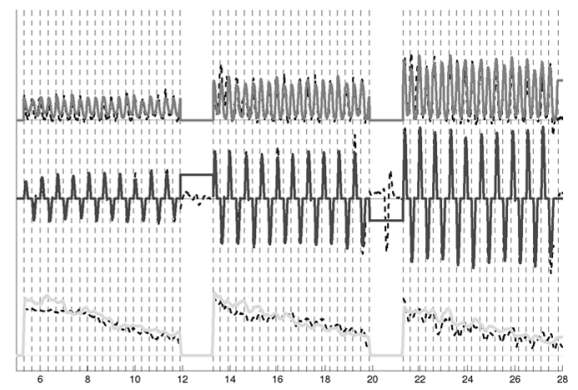

(f)

Fig. 10. Rendering results of bowing parameter contours. From top to bottom: bow force ( 0.02 N/unit), bow velocity (cm/s), and bow-bridge distance $(0.04$ $\mathrm{cm} /$ unit). Horizontal thin lines correspond to zero levels, solid thick curves represent rendered contours, and dashed thin curves represent acquired contours. Vertical dashed lines represent note onset/offset times (seconds). (a) Détaché articulation, (b) Legato articulation, (c) Alternating détaché and legato articulation, (d) Saltato articulation, (e) Staccato articulation, (f) Piano, mezzoforte, and forte dynamics for staccato articulation.

presented in this work (including the bow planning algorithm), are shown in Fig. 10. In order to examine the results, we used existing scores in the corpus as an input to the rendering algorithm. By using note onset/offset times of the recorded performances instead of the nominal times, it is possible to visually compare the rendered and recorded contours.

Due to an unbalanced database note distribution, the number of notes belonging to some of the note classes was much smaller than for other classes, thus causing some performance context sub-clusters to contain very few curve parameter vectors. In these cases, a specific treatment is carried out, being opposite to the already implemented policy of iteratively reducing the number of performance context sub-clusters (see Section IV-E). The solution, designed to artificially overcome variance problems, consists of decomposing covariance matrices of less populated sub-clusters into eigenvalues and eigenvectors, and scaling eigenvalues by a factor inversely proportional to a relative measure of the number of vectors used when estimating the distribution parameters (see Section IV-C).

\section{B. Physical Model-Based Sound Synthesis}

Synthetic contours of bowing control parameters have been used for driving a modified version of the Cook and Scavone Synthesis Toolkit in C++ (STK) [36] implementation of Smith's digital waveguide bowed-string physical model [23], sketched in Fig. 11. A single string model with a low-pass one-pole implementation of the loss filter has been chosen as a proof-of-concept physical model for our bowing control model. In order to simulate the body impulse response, we convolved the output signal coming from the physical model with different impulse

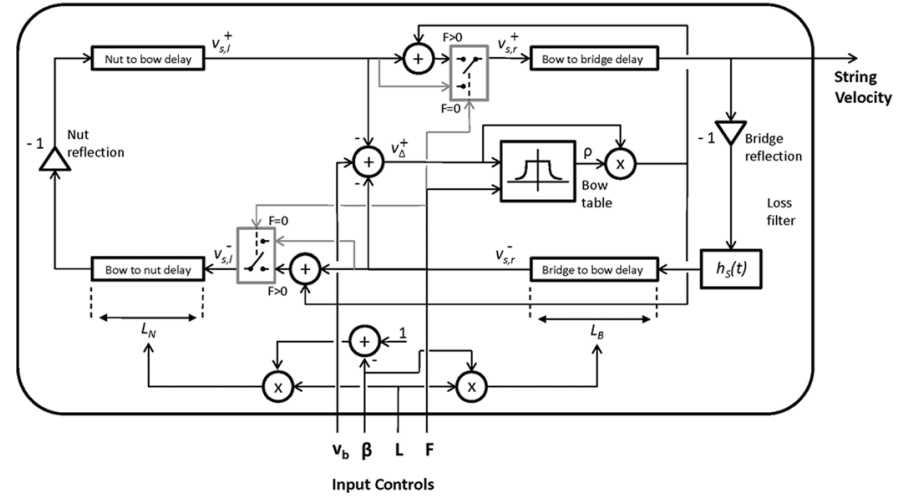

Fig. 11. Smith's digital waveguide bowed-string physical model. Performance controls include bowing control parameters (bow velocity $v_{b}$, bow force $F_{b}, \beta$ ratio), and delay line length $L$ (corresponding to string length for a given pitch). Switches were added as a practical solution for easily incorporating off-string bowing conditions.

responses computed through different methods [37], including the body filter response estimated as outlined in Section II-C. The exact magnitude measured with the pickup has not been determined, but observations made it apparent that the signal is closer to the force of the string on the bridge than to the string velocity itself. Since the output of the physical model is the string velocity, improved results were obtained when using an impulse response extracted by measuring the string velocity with a magnet below the bridge end of the string. Sound synthesis examples are available online. ${ }^{9}$

\footnotetext{
${ }^{9} \mathrm{http}: / /$ www.dtic.upf.edu/emaestre/gestureModels/bowed/violin/spm.zip
} 
1) Model Description: In Fig. 11, the right delay line pair carries left-propogating and right-propogating velocity wave samples $v_{\mathrm{sr}}^{+}$and $v_{\mathrm{sr}}^{-}$, respectively, which sample the traveling wave components within the string to the right of the bow, and similarly for the section of string to the left of the bow. The " + " superscript refers to waves traveling into the bow. String velocity $v_{s}$ at any point is obtained by adding a left-propogating velocity sample to the right-propogating velocity sample immediately opposite in the other delay line (this occurs in Fig. 11 at the bowing point). The loss filter represents the losses at the bridge, bow, nut, or finger-terminations, and the total attenuation from traveling back and forth on the string. The bow-string nonlinear interaction is defined as a function of the differential velocity $v_{\Delta}^{+}$(bow velocity $v_{b}$ minus string velocity $v_{s}$ ) and the bow force $F_{b}$ which, by modifying the shape of the bow table function, provides the real-valued reflection coefficient $\rho$. The coefficient $\rho$ defines the proportion between waves being reflected by and traveling through the bow. Bow-bridge distance modifies the proportion of the delay line length $L$ (corresponding to a given pitch) that goes into the bow-nut delay line length $L_{N}$ and the bow-bridge delay line length $L_{B}$.

2) Calibration Issues: Digital waveguide physical model calibration represents a challenging problem not addressed in this paper. We ignored left-hand articulation-related control parameters (e.g., vibrato), so pitch transitions were considered to be instantaneous (implying sudden changes on the delay line length $L$ ), and string terminations were each represented by a reflection coefficient $\rho=-1$. For the loss filter of the single-string model, gain value and pole positioning were manually set by listening to synthesized sound.

While both bow velocity $v_{b}$ and $\beta$ ratio were used directly in the model, bow force $F_{b}$ was used to configure the shape of bow table. The bow table was defined by a breakaway differential velocity $v_{\Delta}^{c}$ and an exponential decaying factor $S$, both depending on $F_{b}$ [23]. Two linear mapping functions $v_{\Delta}^{c}=f\left(F_{b}\right)$ and $S=f\left(F_{b}\right)$ were manually tuned also by listening to synthesis results. In order to approximate off-string bowing conditions (e.g., saltato articulation), bow-string interaction (represented by the bow table) is bypassed when bow force becomes non-positive (see the switches in Fig. 11). This implies left and right delay lines to be connected, leading to a damped oscillation between successive saltato bow strokes. A physical modeling solution to this would instead need an additional calibration function by which the bow table would smoothly morph to zero (no waves reflected by the bow) as the force approaches zero. In order to overcome the calibration difficulties brought by such additional function (recall that calibration is not addressed here), a more practical solution was achieved with the aforementioned switches.

\section{Sample-Based Sound Synthesis}

The obtained results on bowing control modeling presented have also been preliminarily applied to sample-based, spectral-domain concatenative sound synthesis. This was done by making use of the same corpus utilized for constructing the contour models. In the sample database, both the contours and their corresponding curve parameter vectors (see Section IV-A) are included as part of the annotations of each sample. Rendered bowing controls are used both during note sample retrieval and during note sample transformation, with the aim to meaningfully improve sample discontinuities and timbre transformation. Sample transformation is performed in the spectral domain to stored pickup audio signals (e.g., note samples), thus avoiding potential problems introduced by body resonances. Assuming the body filtering effect to be linear, the resulting audio signal is convolved with a body impulse response that was previously obtained by deconvolution as briefly described in Section II-C. Sound synthesis examples are available online ${ }^{10}$.

1) Sample Retrieval: As a first step, a sample candidate list $\gamma_{i}$ is generated for each $i$ th note in the input score by making use of different sample annotations: matching articulation, bow direction, played string, bow context, and silence context (see Section III-A). Then, sample retrieval is performed through an optimal path search in a similar fashion as presented in [38] after [39] (again making use of dynamic programming). In this work, the main novelty is the introduction of a measure of distance between target bowing control parameters and sample bowing control parameters, therefore enabling the search for samples by accounting for bowing parameter contours. The best sequence $\gamma^{*}$ of $N$ candidate indexes is found by minimizing a total cost path $C$

$$
\begin{aligned}
\gamma^{*} & =\left[\gamma_{1}^{*} \cdots \gamma_{N}^{*}\right] \\
& =\underset{\gamma}{\operatorname{argmin}} C(\gamma) .
\end{aligned}
$$

Three main sub-costs are computed for each of the $N$ notes in the sequence: a perceptual distance $\operatorname{cost} C_{p}$, a bowing control distance $\operatorname{cost} C_{g}$, and a sample continuity $\operatorname{cost} C_{c}$. This is expressed as

$C(\gamma)=\sum_{i=1}^{N} \omega_{p} C_{p}\left(\gamma_{i}\right)+\omega_{g} C_{g}\left(\gamma_{i}\right)+\sum_{i=1}^{N-1} \omega_{c} C_{c}\left(\gamma_{i}, \gamma_{i+1}\right)$

where $\omega_{p}, \omega_{g}, \omega_{c}$ respectively represent the manually adjusted weights that are applied to $C_{p}, C_{g}$, and $C_{c}$. Note that the continuity cost $C_{c}$ is computed for every pair of successive notes.

The perceptual distance cost $C_{p}$ between target note and candidate sample is computed by comparing note duration, fundamental frequency, annotated dynamics, and fundamental frequency intervals of preceding and following notes.

The bowing control distance cost $C_{g}$ is computed as the Mahalanobis distance $D_{M}\left(p_{f}, p_{s}\right)$ between the curve parameter vector $p_{f}$ (see Section V.C) and the annotated bowing control parameter vector $p_{s}$ of the candidate sample. This can be written as

$$
C_{g}=D_{M}\left(p_{f}, p_{s}\right)=\sqrt{\left(p_{f}-p_{s}\right)^{T \Sigma^{*-1}\left(p_{f}-p_{s}\right)}}
$$

where $\Sigma^{*}$ corresponds to the mixed covariance matrix that was used for obtaining $p_{f}$ (see Section V-A).

The continuity cost $C_{c}$ between $i$ th and $(i+1)$ th notes is set as a penalty for encouraging the algorithm to retrieve samples that appear contiguous in the database.

2) Sample Transformation: Fundamental frequency and duration mismatches between input score values and retrieved

\footnotetext{
${ }^{10}$ http://www.dtic.upf.edu/ emaestre/gestureModels/bowed/violin/ssb.zip
} 
sample annotations are transformed in the spectral domain by means of an implementation of the phase-locked vocoder techniques described in [40] and [41]. In particular, time-scaling is applied non-uniformly along each sample so that sustain segments carry most of duration transformation.

Retrieved samples are framewise transformed in the spectral domain by taking into consideration the differences found between rendered and retrieved contours. Both the rendered contours and the retrieved contours (the latter resulting from the concatenation of the contours of retrieved samples) are used to control a neural network-based violin timbre model that is able to predict spectral envelopes of the harmonic and residual content, given the instantaneous values of bow velocity, bow force, and bow-bridge distance. Two pairs of spectral envelopes (harmonic and residual) are consequently obtained for each frame, respectively corresponding to the predictions obtained from instantaneous values of rendered parameters and from those of retrieved samples. Predicted spectral envelopes are then subtracted for each frame, resulting in a differential envelope for the harmonic content and another for the residual content. The resulting differential envelopes are used as a time-varying filter for modifying the spectral content (harmonic and residual) of retrieved samples. Sample transformations that are meaningful to bowing control context are thereby achieved, causing timbre continuity to significantly improve, especially in the concatenation surroundings. Details concerning the timbre model, which was trained by feeding it with audio data aligned to bowing control data (as it appears in the database used for synthesis), can be found in [28], and [32].

\section{CONCLUSION}

In this paper, we have presented an approach for bowing control modeling in the context of violin sound synthesis. The framework is based on a method for automatically characterizing contours of relevant bowing control parameters acquired from real violin performance. Contours are represented by concatenating cubic Bézier curve segments following a scheme that is structured at note-level. An analysis-synthesis statistical model of bowing control contours is built on top of obtained curve parameters. The model is used for rendering bowing parameter contours (bow velocity, bow force, and bow-bridge distance) from annotated input scores. Synthetic bowing parameter contours are successfully used for driving violin sound generation using a digital waveguide-based physical model and a sample-based spectral-domain concatenative synthesis framework.

It represents a difficult task to quantitatively assess the validity of the proposed bowing control model. In our implementation, we tested a significant number of model parameter configurations (e.g., grammar definitions, clustering parameters), searching for an optimal setting by comparing rendering results to acquired data and by listening to synthesized sound. An unevenly distributed note database led to some inconsistencies in the statistical model that had to be artificially compensated by scaling down the eigenvalues of some normal distributions. Also, missing recordings for notes in particular performance contexts (especially concerning starting bow position) kept the bow planning algorithm from being fully consistent. With a larger database, a deeper analysis of the different choices for the model parameters would both enhance population of clusters, and serve as a better test-bed for quantitatively validating the performance of the bow planning model.

Several extensions are possible, such as adding more note articulations, considering left-hand controls, or adding a stochastic component on top of rendered contours. An extended database including performance from different musicians playing different styles would further validate this modeling framework. Approaches for automatic grammar definition would be of great value when extending this framework to other excitation-continuous instruments. Additionally more violin use-cases could be studied. For example, automatic performance annotation could prove to be useful for expressiveness or style analysis based on studying choices of articulations, bow directions, etc. Complementary perspectives for modeling motion (e.g., kinematics) may also be considered to be a roadmap for further research.

For the case of physical model-based sound synthesis, we observed that the resulting sound impressively improved in terms of realism and naturalness even though a very simplified, non-calibrated digital waveguide model was used. Incorporating more complex physical models has a high potential for further improving the sound. Although we did not primarily devote our research to calibrating the physical model, an automatic calibration procedure (based on spectral-matching and using acquired bowing control and audio data) is currently under development.

Regarding sample-based sound synthesis, the resulting sounds also demonstrated a high degree of realism. Although some of the expressive features of database samples were retained and a simple vibrato model was applied, an improvement of the feeling of timbral continuity (especially around sample boundaries) was apparent. Nevertheless, possible discontinuities occur mainly due to sample selection errors or sample transformation limitations. Thus, fine adjustments of sample selection weighting and tuning of the timbre model will improve results in future implementations.

Generally, the instrumental control modeling framework presented in this study points to future directions in musical instrument sound synthesis. As appropriate techniques for measuring control parameters become available, emulating playing habits in human performance may become feasible, thereby bringing instrumental sound synthesis to a higher level. Current samplebased synthesis techniques may already start to take benefit from the ideas of performer-instrument decoupling as introduced in this work. The combination of automatic control rendering with control-based spectral-domain sample transformation may provide a degree of controllability close to that of physical models. For the case of physical-models, next generation synthesizers able to represent the most complex sound production mechanisms will nevertheless require a control model in order to exploit the great control flexibility derived from their nature.

\section{ACKNOWLEDGMENT}

The authors would like to thank J. Abel, J. O. Smith III, and A. Zymnis for inspiring discussion and fruitful advice. 


\section{REFERENCES}

[1] C. Cadoz and C. Ramstein, "Capture, representation and composition of the instrumental gesture," in Proc. 1990 Int. Computer Music Conf., Glasgow, U.K., 1990, pp. 53-56.

[2] E. Maestre, "Coding instrumental gestures: Towards automatic characterization of instrumental gestures in excitation-continuous musical instruments," DEA Doctoral pre-thesis work, Universitat Pompeu Fabra, Barcelona, Spain, 2006.

[3] A. Askenfelt, "Measurement of bow motion and bow force in violin playing," J. Acoust. Soc. Amer., vol. 80, no. 4, pp. 1007-1015, 1986.

[4] A. Askenfelt, "Measurement of the bowing parameters in violin playing. II. bow-bridge distance, dynamic range, and limits of bow force," J. Acoust. Soc. Amer., vol. 86, no. 2, pp. 503-516, 1989.

[5] J. A. Paradiso and N. A. Gershenfeld, "Musical applications of electric field sensing," Comput. Music J., vol. 21, no. 2, pp. 69-89, 1997.

[6] D. Young, "A methodology for investigation of bowed string performance through measurement of violin bowing technique," Ph.D. dissertation, Mass. Inst. of Technol., Cambridge, U.K., 2007.

[7] N. Rasamimanana, E. Fléty, and F. Bevilacqua, "Gesture analysis of violin bow strokes," Lecture Notes in Comput. Sci., vol. 3881, pp. 145-155, 2006.

[8] E. Schoonderwaldt, N. Rasamimanana, and F. Bevilacqua, "Combining accelerometer and video camera: reconstruction of bow velocity profiles," in Proc. 2006 Int. Conf. New Interfaces for Musical Expression, Paris, France, 2006, pp. 200-203.

[9] M. Demoucron, "On the control of virtual violins: Physical modelling and control of bowed string instruments," Ph.D. dissertation, Univ. Pierre et Marie Curie (Paris 6) and the Royal Inst. of Technol., KTH, Stockholm, 2008.

[10] M. Demoucron, A. Askenfelt, and R. Caussé, "Measuring bow force in bowed string performance: Theory and implementation of a bow force sensor," Acta Acustica United with Acustica, vol. 95, no. 4, pp. 718-732, 2009.

[11] E. Guaus, J. Bonada, A. Pérez, E. Maestre, and M. Blaauw, "Measuring the bow pressing force in a real violin performance," in Proc. 2007 Int. Symp. Musical Acoust., Barcelona, Spain, 2007.

[12] E. Maestre, J. Bonada, M. Blaauw, E. Guaus, and A. Pérez, "Acquisition of violin instrumental gestures using a commercial emf device," in Proc. 2007 Int. Comput. Music Conf., Copenhagen, Denamrk, 2007, vol. 1, pp. 386-393.

[13] E. Schoonderwaldt and M. Demoucron, "Extraction of bowing parameters from violin performance combining motion capture and sensors," J. Acoust. Soc. Amer., vol. 126, no. 5, pp. 2695-2708, Nov. 2009.

[14] N. H. Rasamimanana, D. Bernardin, M. Wanderley, and F. Bevilacqua, "String bowing gestures at varying bow stroke frequencies: A case study," in Advances in Gesture-Based Human-Computer Interaction and Simulation, ser. Lecture Notes in Computer Science. New York: Springer Verlag, 2009, vol. 5085, pp. 216-226.

[15] D. Young, "Classification of common violin bowing techniques using gesture data from a playable measurement system," in Proc. 2008 Int. Conf. New Interfaces for Musical Expression, Genova, Italy, 2008.

[16] N. H. Rasamimanana and F. Bevilacqua, "Effort-based analysis of bowing movements: evidence of anticipation effects," J. New Music Res., vol. 37, no. 4, pp. 339-351, 2008.

[17] C. Chafe, "Simulating performance on a bowed instrument," Stanford Univ., Stanford, CA, CCRMA Tech. Rep. STAN-M48, 1988.

[18] D. Jaffe and J. Smith, "Performance expression in commuted waveguide synthesis of bowed strings," in Proc. 1995 Int. Comput. Music Conf., Banff, AB, Canada, 1995.

[19] E. Rank, "A player model for MIDI control of synthetic bowed strings," in Proc. DIDEROT Forum on Math. and Music, Vienna, Austia, 1999.

[20] D. Escudero, V. Cardeñoso, and A. Bonafonte, "Corpus-based extraction of quantitative prosodic parameters of stress groups in spanish," in Proc. 2002 Int. Conf. Acoust., Speech, Signal Process., Orlando, FL, 2002.

[21] B. Battey, "Bézier spline modeling of pitch-continuous melodic expression and ornamentation," Comput. Music J., vol. 28, no. 4, pp. 25-39, 2004.

[22] E. Maestre, J. Bonada, and O. Mayor, "Modeling voice articulation gestures in singing voice performance," in Preprints of the Audio Eng,. Soc. 121 st Conv., San Francisco, CA, 2006.

[23] J. O. Smith, Physical Audio Signal Processing, December 2008 Edition. Stanford, CA: [Online]. Available: http://ccrma.stanford.edu/ jos/pasp/, accessed 2009, online book
[24] D. Young and S. Serafin, "Playability evaluation of a virtual bowed string instrument," in Proc. 2006 Int. Conf. New Interfaces for Musical Expression, Montréal, QC, Canada, 2003, pp. 104-108.

[25] S. Serafin, "The sound of friction: Real-time models, playability and musical applications," Ph.D. dissertation, Stanford Univ., Stanford, CA, 2004.

[26] B. Schoner, C. Cooper, and N. Gershenfeld, "Cluster weighted sampling for syntheis and cross-synthesis of violin family instruments," in Proc. 2000 Int. Comput. Music Conf., Berlin, Germany, 2000.

[27] E. Lindemann, "Music synthesis with reconstructive phrase modeling," IEEE Signal Process. Mag., vol. 24, no. 2, pp. 80-91, 2007.

[28] A. Pérez, J. Bonada, E. Maestre, E. Guaus, and M. Blaauw, "Combining performance actions with spectral models for violin sound transformation," in Proc. 2007 Int. Congr. Acoust., Madrid, Spain, 2007.

[29] E. Maestre, "Data-driven statistical modeling of violin bowing gesture parameter contours," in Proc. 2009 Int. Computer Music Conf., Montréal, QC, Canada, 2009.

[30] E. Guaus, M. Blaauw, J. Bonada, E. Maestre, and Pérez, "A calibration method for accurately measuring bow force in real violin performance," in Proc. 2009 Int. Comput. Music Conf., Montréal, QC, Canada, 2009.

[31] E. Maestre, "Modeling instrumental gestures: An analysis/synthesis framework for violin bowing," Ph.D. dissertation, Univ. Pompeu Fabra, Barcelona, Spain, 2009.

[32] A. Pérez, "Enhancing spectral synthesis techniques with performance gestures using the violin as a case study," Ph.D. dissertation, Univ. Pompeu Fabra, Barcelona, Spain, 2009.

[33] A. J. Viterbi, "Error bounds for convolutional codes and an asymptotically optimum decoding algorithm," IEEE Trans. Inf. Theory, vol. IT-13, no. 2, pp. 260-269, Apr. 1967.

[34] M. Karjalainen, V. Välimäki, H. Penttinen, and H. Saastamoinen, "DSP equalization of electret film pickup for the acoustic guitar," J. Audio Eng. Soc., vol. 48, no. 12, pp. 1183-1193, 2000.

[35] J. B. MacQueen, "Some methods of classification and analysis of multivariate observations," in Proc. 5th Berkeley Symp. Math. Statist. Probab., 1967, pp. 281-297.

[36] P. Cook and G. Scavone, The Synthesis Toolkit in C++, 2007 Edition [Online]. Available: http://ccrma.stanford.edu/software/stk/ accessed 2009, online software. 17

[37] P. Cook and D. Trueman, "A database of measured musical instrument body radiation impulse responses, and computer applications for exploring and utilizing the measured filter functions," in Proc. 1998 Int. Symp. Musical Acoust., Leavenworth, WA, 1998.

[38] E. Maestre, R. Ramírez, S. Kersten, and X. Serra, "Expressive concatenative synthesis by reusing samples from real performance recordings," Comput. Music J., vol. 33, no. 4, pp. 23-42, 2009.

[39] D. Schwarz, "Data-driven concatenative sound synthesis," Ph.D. dissertation, IRCAM Centre Popidou, Univ. Pierre et Marie Curie (Paris 6), Paris, France, 2004.

[40] J. Laroche, "Frequency-domain techniques for high-quality voice modifications," in Proc. 2003 Int. Conf. Digital Audio Effects, London, U.K., 2003.

[41] J. Bonada, "Automatic technique in frequency domain for near-lossless time-scale modification of audio," in Proc. 2000 Int. Comput. Music Conf., Berlin, Germany, 2000.

[42] A. Pèrez, "Enhancing spectral synthesis techniques with performance gestures using the violin as a case study," Ph.D. dissertation, Univ. Pompeu Fabra, Barcelona, Spain, 2009.

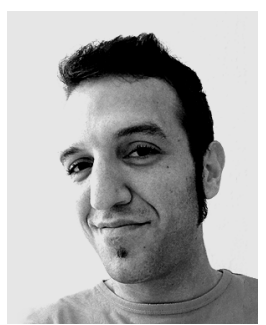

Esteban Maestre was born in 1979. He received the B.Sc. and M.Sc. degrees in electrical engineering from the Universitat Politècnica de Catalunya, Barcelona, Spain, in 2004, and the Ph.D. degree in computer science and digital communication from the Universitat Pompeu Fabra, Barcelona, in 2009.

$\mathrm{He}$ worked as a Junior Researcher at Philips Research Laboratories, Aachen, Germany, during 2003 and 2004, and was a Visiting Researcher at the Center for Computer Research in Music and Acoustics, Stanford University, Stanford, CA, during 2008. Since 2003, he has been a Lecturer and a Researcher both at Universitat Politècnica de Catalunya and at Universitat Pompeu Fabra. He is currently a Researcher (Music Technology Group) and a Lecturer (Department of Information and Communication Technologies) at Universitat Pompeu Fabra. His research interests include sound analysis and synthesis, speech processing, and gesture modeling. 


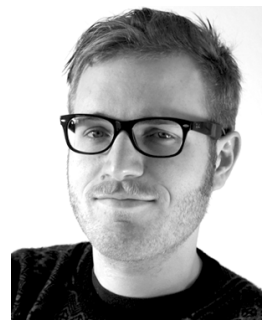

Merlijn Blaauw was born in 1980. He received the M.A. degree in sound and music technology from the Hogeschool voor de Kunsten Utrecht, Utrecht, The Netherlands, in 2004.

He is currently a member of the Music Technology Group, Universitat Pompeu Fabra, Barcelona, Spain, where he has been involved in several collaborative projects with Yamaha Corp. Japan. Most of his work has been focused on the development of concatenative, sample-based synthesis techniques applied to singing voice and the violin.

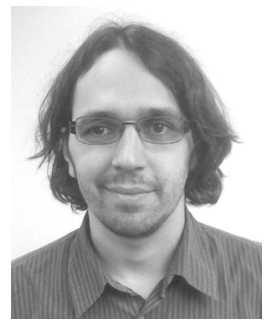

Jordi Bonada was born in 1973. He received the M.Sc. degree in electrical engineering from the Universitat Politècnica de Catalunya, Barcelona, Spain, in 1997, and the Ph.D. degree in computer science and digital communications from Universitat Pompeu Fabra, Barcelona, Spain, in 2009.

Since 1996, he has been a Lecturer at the Universitat Pompeu Fabra and a Researcher at the Music Technology Group of the same university while leading several collaboration projects with Yamaha Corp. Japan. In 2003, he was a Visiting Researcher at the Royal Institute of Technology, Stockholm, Sweden. He is mostly interested in the field of spectral-domain audio signal processing, with focus on time-scaling and singing voice modeling and synthesis.

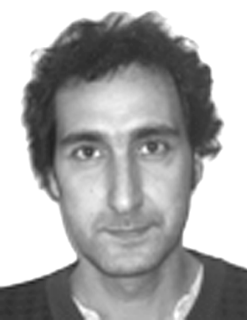

Enric Guaus was born in 1974. He received the $\mathrm{Ph} . \mathrm{D}$. degree in computer science and digital communications from the Universitat Pompeu Fabra, Barcelona, Spain, in 2009 with a dissertation on automatic music genre classification.

$\mathrm{He}$ is a Researcher in sound and music computing at the Music Technology Group (MTG), Universitat Pompeu Fabra (UPF), Barcelona, Spain. His research interests cover music information retrieval and human interfaces for musical instruments. He is an Assistant Professor in audio and music processing at UPF and a Lecturer in acoustics, electronics, and computer science at the Escola Superior de Música de Catalunya (ESMUC).

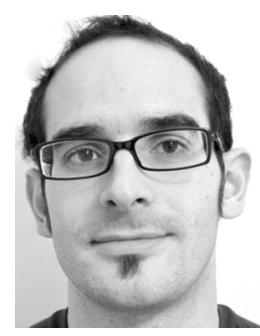

Alfonso Pérez was born in 1977. He received the $\mathrm{Ph} . \mathrm{D}$. degree in music technology from the Universitat Pompeu Fabra, Barcelona, Spain.

$\mathrm{He}$ is a Researcher at the Music Technology Group, Universitat Pompeu Fabra, where he also teaches computer programming. He studied computer science and violin. His main research interest is sound synthesis of musical instruments with a special focus on the violin. 